\title{
Recent Progress in the Synthetic Assembly of 2-Cyclopentenones
}

\author{
David J. Aitken, ${ }^{\text {a }}$ Hendrik Eijsberg, ${ }^{\text {a,b }}$ Angelo Frongia, ${ }^{\mathrm{b}}$ Jean Ollivier, ${ }^{\mathrm{a}}$ Pier Paolo Piras ${ }^{\mathrm{b}}$ \\ a Laboratoire de Synthèse Organique \& Méthodologie, ICMMO (CNRS UMR 8182), Université Paris Sud, 15 rue Georges Clemenceau, \\ 91045 Orsay cedex, France \\ Fax +33(1)69156278; E-mail: david.aitken@u-psud.fr \\ b Dipartimento di Scienze Chimiche e Geologiche, Università degli studi di Cagliari, Complesso Universitario di Monserrato, S.S. 554, \\ Bivio per Sestu, 09042 Monserrato, Cagliari, Italy \\ Received: 09.07.2013; Accepted after revision: 21.08.2013
}

\author{
Abstract: An overview of the most important synthetic strategies \\ currently available for the preparation of cyclopent-2-enones is pre- \\ sented and illustrated with recent applications. \\ 1 Introduction \\ 2 Multicomponent Ring Assembly \\ 3 Cyclizations \\ 4 Transformations of Existing Cyclic Systems \\ 5 Miscellaneous Methods \\ 6 Conclusions
}

Key words: cyclopentenones, cyclization, carbocycles, ring closure, rearrangement, annulation

\section{$1 \quad$ Introduction}

\section{Scope of this Review}

2-Cyclopentenones are a frequently encountered class of cyclic enone. They feature in many areas of organic chemistry and serve as benchmark substrates for numerous chemical transformations, and natural product structures containing a 2-cyclopentenone molecular feature are ubiquitous.

Some of the synthetic routes to the title compounds have been reviewed periodically ${ }^{1}$ (see also specific sections), but a global appraisal has not appeared for some time. ${ }^{2}$ This review covers the literature over the last decade or so, and it endeavors to provide an overview of the most commonly used synthetic approaches for assembling the eponymous core feature from unrelated precursors. It is structured according to the strategy by which the cyclic enone feature is created, rather than according to any particular type of derivative or substitution pattern. New approaches as well as new results using established ones are considered equally.

The numerous methods available for the generation of 2cyclopentenones by $\alpha, \beta$-elimination reactions of appropriately functionalized cyclopentanone precursors fall outside of the scope of this review, as do the vast array of oxidations of cyclopentenes, 2-cyclopentenols and cyclopentanones. These limitations notwithstanding, there are a

SYNTHESIS 2014, 46, 0001-0024

Advanced online publication: 21.11.2013

DOI: 10.1055/s-0033-1340414; Art ID: SS-2013-E0467-R

(C) Georg Thieme Verlag Stuttgart $\cdot$ New York considerable number of ways in which the target ring system can be created from acyclic precursors, in either intermolecular or intramolecular mode. Most of the possible disconnection strategies have been examined, and it is important to recognize that for any given target 2-cyclopentenone, there may be several convenient approaches available. The main approaches for ring construction are summarized graphically in Figure 1.
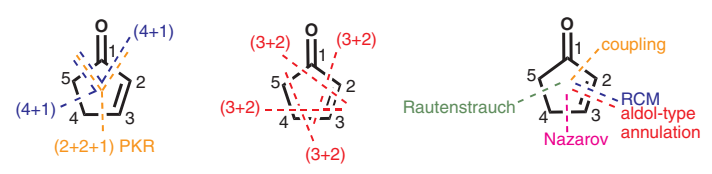

Figure 1 The main ring-construction strategies for 2-cyclopentenone synthesis, showing the atom connectivities made during ring assembly (left and center) and cyclization approaches (right). These and other strategies are covered in this review.

Useful procedures based on the transformation of existing cyclic structures also exist, as do some miscellaneous methods; these will be treated towards the end of this review.

The 'ideal' choice of synthetic route depends both on the specific features in the target skeleton, such as the presence of sensitive functional groups or stereogenic centers, and on contextual constraints, such as the employment (or the preclusion) of metals, heat, particular solvents, and so on. Control of the relative configuration of 4,5-disubstituted 2-cyclopentenones can be achieved either by using a precursor in which those chiral centers are already established, or by using an approach in which these centers are created in a diastereoselective fashion, such as the Nazarov or related cyclizations. The preparation of nonracemic 4- and/or 5-substituted 2-cyclopentenones frequently relies on the use of nonracemic chiral substrates. The use of chiral auxiliaries during the ring-creation process has allowed some asymmetric syntheses to be performed, but catalytic enantioselective syntheses are rare at present; indeed this might constitute an area of particular attention for future developments.

Applications of the synthetic approaches reviewed herein have been selected for illustrative purposes as best as possible, but the quantity of work conducted in the area and the structural diversity of the molecular targets make it unfeasible to present an exhaustive list of synthetic applications here. 


\section{Biographical Sketches}
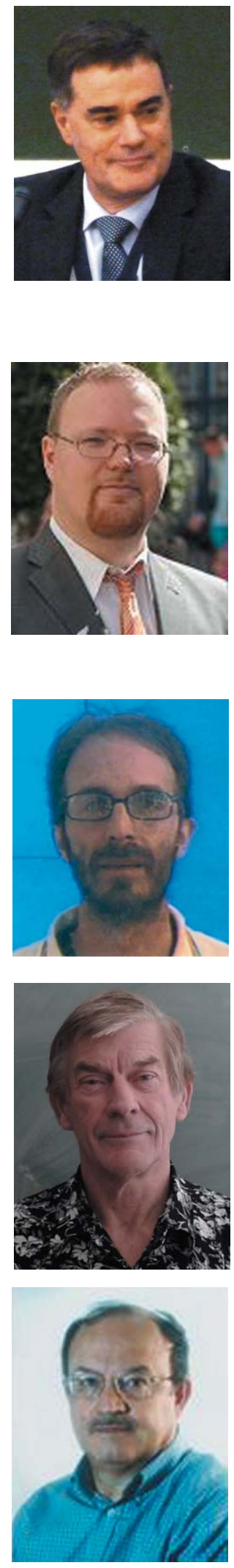

David J. Aitken was born in 1963 and studied chemistry at the University of Strathclyde (Glasgow), obtaining his $\mathrm{PhD}$ in 1986, under the supervision of Prof. H. C. S. Wood and Prof. C. J. Suckling. After a twoyear post-doctoral appointment with Prof. H.-P. Husson at the ICSN (Gif-

Hendrik Eijsberg studied at the Ecole Nationale Superieure de Chimie de Paris (France) and carried out undergraduate work on the asymmetric conjugate addition of organoboron compounds catalyzed by rhodium-diene complexes in the group of Prof. J.-P. Genet and Dr. S. Darses. In

Angelo Frongia was born in Cagliari (Italy) in 1973. He graduated and received his $\mathrm{PhD}$ degree in Organic Chemistry from the University of Cagliari under the supervision of Prof. P. P. Piras. Following collabora-

Jean Ollivier obtained his $\mathrm{PhD}$ degree in 1982 at the University Paris-Sud (Orsay) under the direction of Dr. J. Salaün, then joined the Centre National de la Recherche Scientifique (CNRS). In 1986 he obtained a Doctorat-ès-Sci-

Pier Paolo Piras received his Laurea in Chemistry at the University of Cagliari (Italy) in 1971 and began his academic career at the same university as Assistant Professor in 1972. He was a postdoctoral fellow (19801981) with Professor C. J. M. Stirling at the University of Bangor (North Wales). In
sur-Yvette) he was appointed CNRS researcher at Descartes University (Paris). In 1998 he became Professor of Organic Chemistry at the University of ClermontFerrand, and in 2006 he transferred to his current position as Professor at the University Paris Sud (Orsay). His research interests, con-

2008, he joined the group of Prof. D. J. Aitken at the University of Paris-Sud (Orsay) where he carried out his $\mathrm{PhD}$ studies on the photochemistry of cyclopentenones and alkenes beyond the [2+2] stage. He collaborated during his $\mathrm{PhD}$ with the group of Prof. P. P. Piras from the University of $\mathrm{Ca}-$

tive post-doctoral research in the group of Prof. D. J. Aitken in the ICMMO, University Paris Sud (Orsay), he joined the academic staff at the University of Cagliari in 2010. He is currently Assistant Professor of Organic

ences degree in Organic Chemistry and then spent a year (1987) as a postdoctoral fellow at the Dyson Perrins Laboratory (Oxford) with Dr. S. G. Davies. He then returned to Orsay and is presently Chargé de Recherche in the ICMMO,

1990-1991 he spent a sabbatical year at the Laboratoire des Carbocycles, University of Paris-Sud (Orsay) working on cyclopropane derivatives with Dr. J. Salaün. Returning to $\mathrm{Ca}$ gliari, he was appointed Associate Professor in 1985, then full Professor of Organic Chemistry in 2001. In ducted in the ICMMO research institute, include the synthesis of functionalized small-ring compounds, particularly unnatural amino acids, as building blocks for foldamers and peptidomimetics, and synthetic organic photochemistry.

gliari (Italy) on an organocatalysis project. $\mathrm{He}$ finished his $\mathrm{PhD}$ in 2012 and joined the group of Professor I. Marek in the Technion Institute (Israel). His research interests now include zirconium-mediated reactions and carbometalation of small rings.

Chemistry at the Faculty of Sciences. His research interests include asymmetric synthesis and development of new synthetic methods based on transformation of strained organic compounds.

University Paris-Sud. His research interests include the chemistry and applications of small-ring compounds implicating stereoand enantioselective reactions, ring expansions and, more recently, organocatalysis.

2009 he was a Visiting Professor in the University of Paris-Sud (Orsay). His primary research interests focus on the synthesis and reactivity of strained carbocycles, the synthesis of natural products, and asymmetric organocatalytic reactions. 


\section{Overview of 2-Cyclopentenone Reactivity}

While the purpose of this review is to relate the main synthetic approaches for the preparation of 2-cyclopentenones, it is useful to present here a brief summary of the chemical reactivity of this molecular core, for two reasons. Firstly, the diversity of chemical transformations that can be carried out thereupon goes some way to explaining the popularity of the system, and secondly, these reactivity features should be kept in mind when planning the synthesis of any particular 2-cyclopentenone derivative. The core structure is highly reactive, with methods available for the modification of every position (Figure 2).

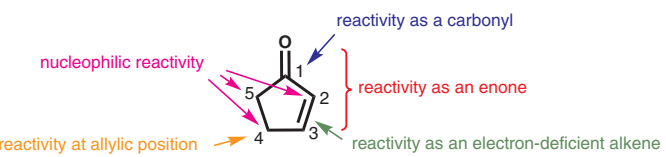

Figure 2 The multiple reactivity profile of the 2-cyclopentenone core structure

At the 1-position, the carbonyl group can react in a typical manner with nucleophiles that give regioselective 1,2-additions to conjugated ketones, such as Luche reduction or addition of organometallic reagents. ${ }^{3}$

The 2-position can be functionalized in a number of ways. The conjugate addition of a weak nucleophile to the 3-position creates an enolate, which reacts at $\mathrm{C} 2$ with carbonyl compounds $^{4}$ or imines ${ }^{5}$ (in Baylis-Hillman-type reactions) or with arylating reagents. ${ }^{6}$ 2-Halocyclopentenones can be prepared similarly ${ }^{7}$ or by other straightforward means $^{8}$ and then engaged in carbon-carbon bond-forming reactions such as palladium-catalyzed couplings ${ }^{9}$ or radical induced additions. ${ }^{10}$ 2-Cyclopentenone-2-boronic acids can also be prepared for cross-coupling reactions. ${ }^{11}$

The 3-position can easily be functionalized via conjugate addition of a variety of nucleophiles, both organic ${ }^{12}$ and heteroatomic, ${ }^{13}$ and these reactions are often amenable to high degrees of enantiomeric control. Heck-type reactions can also be carried out on this position. ${ }^{14}$ The tandem sequence of nucleophilic attack at $\mathrm{C} 3$ followed by electrophilic capture of the intermediate enolate at $\mathrm{C} 2$ is an elegant route to double functionalization. ${ }^{15}$

The 4-position can be brominated with $\mathrm{N}$-bromosuccinimide, ${ }^{16}$ which opens the way to further functionalization at this position. ${ }^{17}$ Vinylogous deprotonation-alkylation procedures generally require a heteroatom substituent at the 3-position, as well as conditions which provide the thermodynamic enolate. ${ }^{18}$

The 5-position can react as a typical $\alpha$ carbon to a carbon$\mathrm{yl}$ function. The generation of a kinetic enolate allows regioselective electrophilic alkylation at $\mathrm{C} 5 .{ }^{19}$ Aldolizations have been described ${ }^{20}$ with recent developments allowing for enantiomeric control. ${ }^{21}$

2-Cyclopentenones are frequent partners in cycloaddition reactions, including photochemical [2+2]-cycloadditions ${ }^{22}$ and Diels-Alder reactions. ${ }^{23}$ Stereoselective cyclopropanations, ${ }^{24}$ aziridinations, ${ }^{25}$ and epoxidations ${ }^{26}$ are also feasible reactions.

\section{$2 \quad$ Multicomponent Ring Assembly}

2-Cyclopentenones can be prepared by assembling two or more components through the formation of at least two new $\sigma$ bonds, generally in a sequential fashion. This is a versatile and often efficient approach for the construction of the target core and it allows for considerable structural diversity. Metal catalysts are often employed.

\section{(2+2+1) Ring Assembly}

The historic example of this type of approach is the Pauson-Khand reaction, in which a cyclopentenone is formed from an alkene and an alkyne in the presence of $\left[\mathrm{Co}_{2}(\mathrm{CO})_{8}\right]$. The generally accepted mechanism (Scheme 1) shows how provision can be made for substituents in all positions. The intermolecular version can be qualified as a $(2+2+1)$ ring assembly. Regioselectivity is an important issue, and is dependent on steric factors: usually $R^{1}$ is larger than $\mathrm{R}^{2}$. Strained or reactive alkenes are privileged; congested alkenes react less well. The intramolecular version - formally a (4+1) assembly, but treated here nonetheless - overcomes a good number of the selectivity issues, and provides a versatile entry to polycyclic skeletons. The reaction has been widely studied and considerable synthetic use has been made thereof. Progress has been reviewed regularly, ${ }^{27}$ with particular attention paid to intramolecular ${ }^{28}$ and catalytic ${ }^{29}$ versions, and a comprehensive monograph has appeared very recently. ${ }^{30}$ Amongst the numerous developments of the PausonKhand reaction, it is worth noting that complexes of metals other than cobalt may serve as catalysts, ${ }^{31}$ while formates or aldehydes can be used as safer CO sources. ${ }^{32}$ The presence of tertiary amine $\mathrm{N}$-oxides is thought to have an accelerating effect, helping in the oxidative removal of one $\mathrm{CO}$ from the alkyne-cobalt complex.

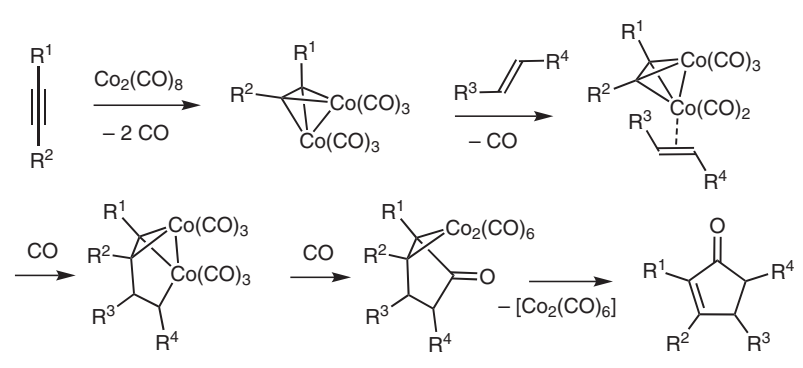

Scheme 1

A few recent applications of the Pauson-Khand reaction are presented here (Scheme 2). Compound $\mathbf{1}$ was subjected to a one-pot reduction-lactonization-Pauson-Khand reaction to give highly functionalized hydropentalenes 2 with high stereoselectivity. ${ }^{33}$ Conditions were found for the Pauson-Khand reaction of unactivated alkene $\mathbf{3}$ with alkyne 4 to provide the 2-cyclopentenone 5 on a multigram scale, as the first step in a total synthesis of the ma- 
rine alkaloids $( \pm)$-axinellamines $\mathrm{A}$ and $\mathrm{B} .{ }^{34}$ In the preparation of structural analogues of the anti-cancer sesquiterpene thapsigargin, an intramolecular rhodiummediated Pauson-Khand reaction was carried out on allene-alkyne 6 to close a seven-membered ring and give product 7 in good yield. ${ }^{35}$ In a recent evaluation of synthetic routes to polycyclic targets, chiral dienediynes $\mathbf{8}$ were found to undergo highly chemoselective PausonKhand reaction in benzaldehyde, which served as the $\mathrm{CO}$ source, using $[\mathrm{Rh}(\operatorname{cod}) \mathrm{Cl}]_{2}$ as the catalyst in the presence of racemic BINAP. High cis-diastereoselectivity (up to $>20: 1$ ) was observed in the products 9 , particularly when bulky substituents were borne adjacent to the chiral center. ${ }^{36}$
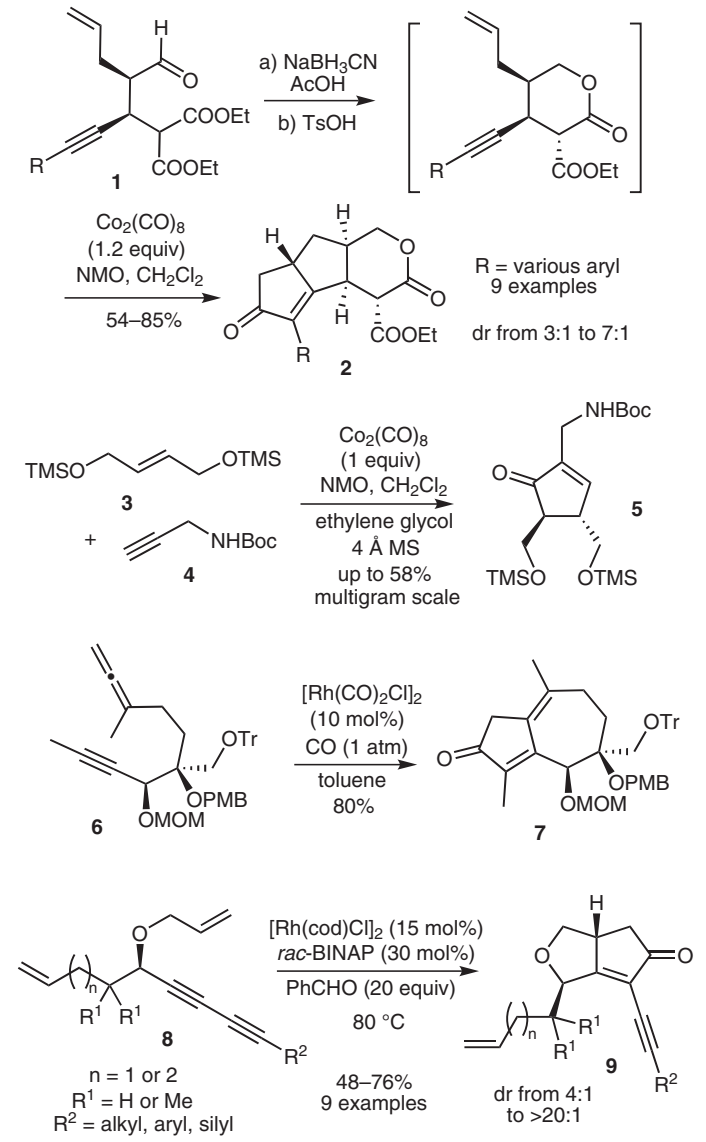

Scheme 2

A $\pi$-allylic precursor can be used instead of the alkyne component. For example, reaction of allyl methyl carbonate with norbornene gave a good yield of the cyclopentenone adduct $\mathbf{1 0}$ with exclusive exo-selectivity (Scheme 3). ${ }^{37}$

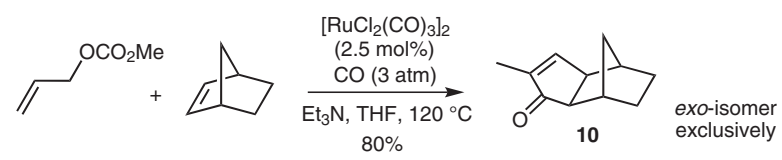

Scheme 3
Reductive $(2+2+1)$ cyclocarbonylations of internal alkynes require more drastic conditions than those habitually employed in the Pauson-Khand reaction, but they have been achieved using a rhodium catalyst in the presence of urea under high carbon monoxide pressure (Scheme 4). A high diastereomeric excess was observed in the products 11, with cis-isomers arising from dialkylalkynes and trans-isomers from diarylalkynes. In the latter cases, yields were lower due to the formation of a lactone byproduct. ${ }^{38}$

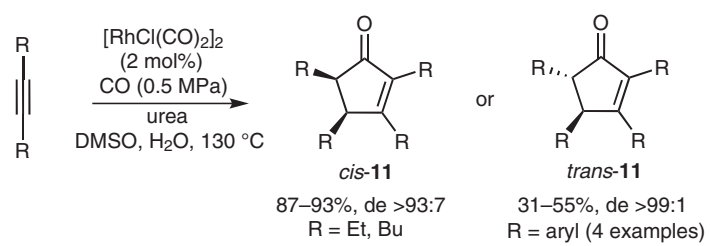

Scheme 4

\section{(3+2) Ring Assembly}

In an effort to overcome some of the limitations of intermolecular Pauson-Khand reactions, a number of $(3+2)$ ring assemblies have been considered. Construction of the five-membered-ring target has been achieved using most of the conceivable disconnections.

Nickel-catalyzed cycloaddition of $\alpha, \beta$-unsaturated phenyl esters 12 with internal alkynes provided trisubstituted 2cyclopentenones 13 (Scheme 5). The regioselectivity varied from poor to excellent, depending on the alkyne used, while terminal alkynes were inefficient substrates. A mechanism was proposed, implicating a $\eta^{3}$-oxaallyl phenoxynickel intermediate. ${ }^{39}$

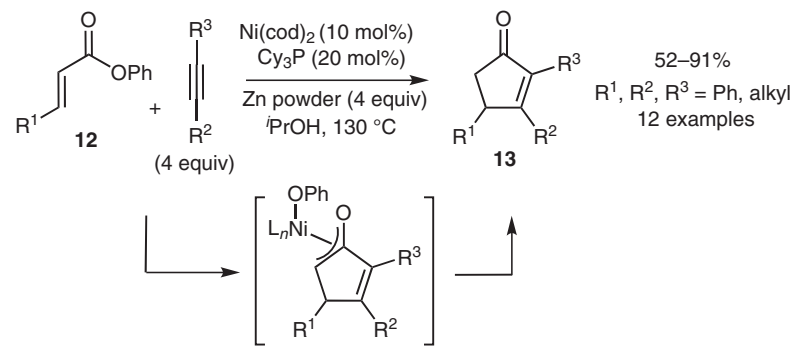

Scheme 5

Nickel-mediated cyclization of alkenyl Fischer carbenes 14 with internal alkynes ${ }^{40}$ provided a wide selection of adducts 15 in a highly regioselective manner (Scheme 6). A recent variation employed a chromium alkynylcarbene $\mathbf{1 6}$ and an alkenyl organolithium 17, both of which were prepared from simple precursors. Again, a variety of substitution patterns were accommodated in the products $\mathbf{1 8}$, and the method can be adapted for enantioselective cyclizations. ${ }^{41}$ Mechanistic models for these transformations were proposed by the authors. 


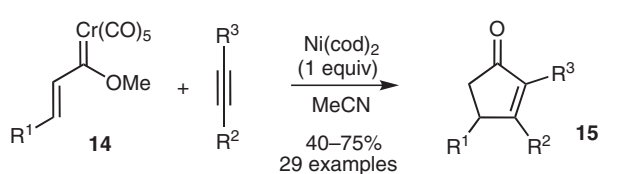

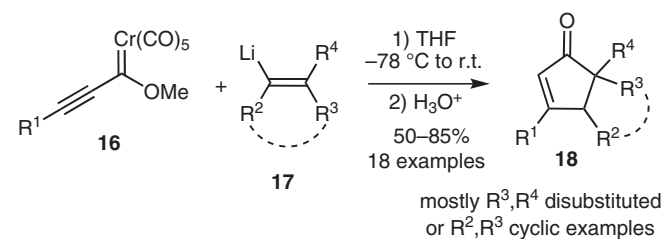

Scheme 6

Vicinal donor-acceptor disubstituted cyclopropanes are convenient precursors for 1,3-dipoles. The Lewis acid mediated reaction of cyclopropanes 19 with silyl ynol ethers gave the $[3+2]$-cycloaddition adducts which spontaneously eliminated ethanol to give the cyclopentadienes 20, which required deprotection with hydrofluoric acid to give the corresponding 2-cyclopentenones 21 (Scheme 7). ${ }^{42}$

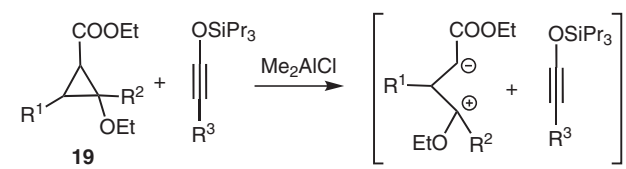

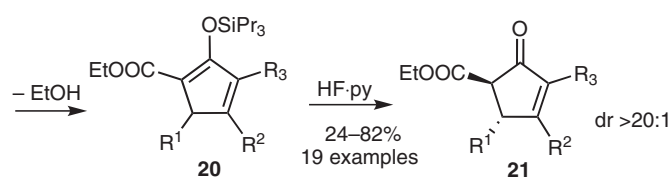

Scheme 7

Zinc chloride promoted a [3+2]-cycloaddition between isoprenyl chloride and methylthio phenylthio ethyne (Scheme 8). The reaction lacked regioselectivity, but the two adducts $\mathbf{2 2}$ and $\mathbf{2 3}$ were separated and transformed easily into the corresponding phenylthio 2-cyclopentenones $\mathbf{2 4}$ and $\mathbf{2 5}$ in good yields. The chemistry of the thioether function was exploited in order to access further derivatives. $^{43}$

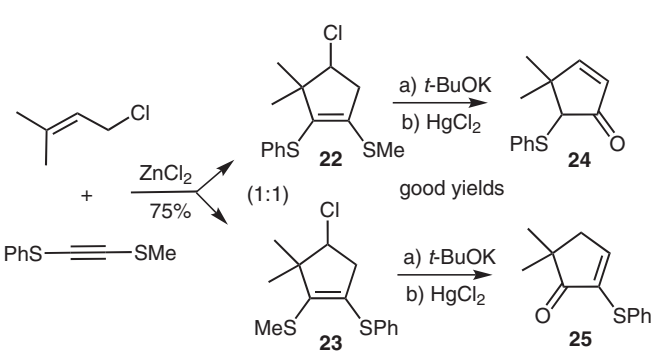

Scheme 8

Interesting results have been observed using allenes as either the two-carbon or three-carbon component in $(3+2)$ assemblies (Scheme 9). Chiral $\alpha$-ethers of allenyl carboxamides $\mathbf{2 6}$ reacted with alkenyllithium reagents to give ad- ducts which, upon addition of acid, gave transient protonated vinyl alkenyl ketones that cyclized to chiral 2cyclopentenones 27 in a conrotatory $4 \pi$-electron process, in Nazarov fashion (vide infra). ${ }^{44}$ It was suggested that this process resulted in axial-to-tetrahedral chirality transfer. $^{45}$ In a complementary fashion, reaction of a chiral lithiated allene $\mathbf{2 8}$ with an $\alpha$-methylcinnamide followed by acidic treatment gave the enantiomerically enriched 2 cyclopentenone derivative $29 .{ }^{46}$
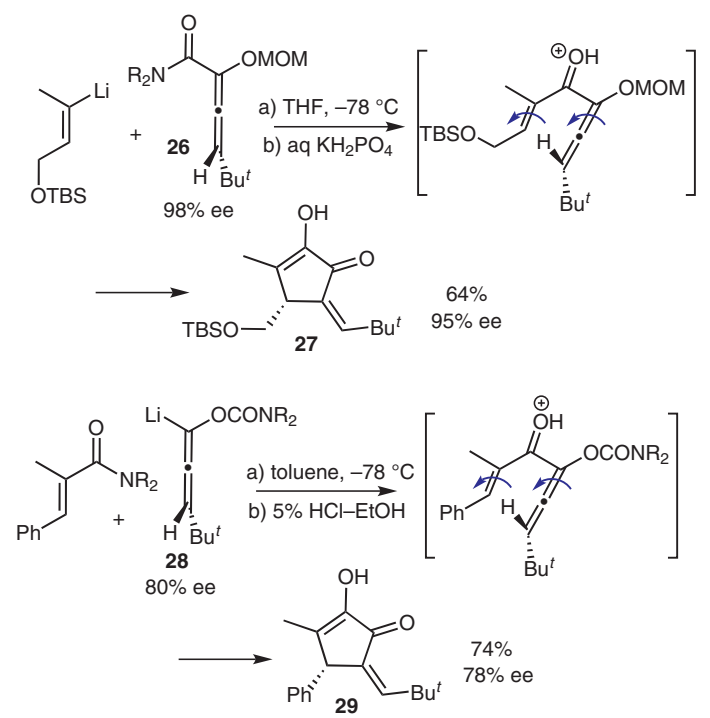

Scheme 9

An organocatalytic iminium ion/N-heterocyclic carbene tandem reaction sequence has been used to combine $\alpha, \beta$ unsaturated aldehydes and $\beta$-keto phenyltetrazolesulfones 30 to give 2,4-disubstituted 2-cyclopentenones 32 in a highly enantioselective manner (Scheme 10). Besides the elegance of the sequential organocatalyzed asymmetric Michael addition-benzoin condensation, the judicious inclusion of a phenyltetrazolesulfone $\left(\mathrm{SO}_{2} \mathrm{PT}\right)$ moiety facilitated a Smiles rearrangement to liberate the target 2cyclopentenones 32; a rational mechanism for this was proposed. ${ }^{47}$
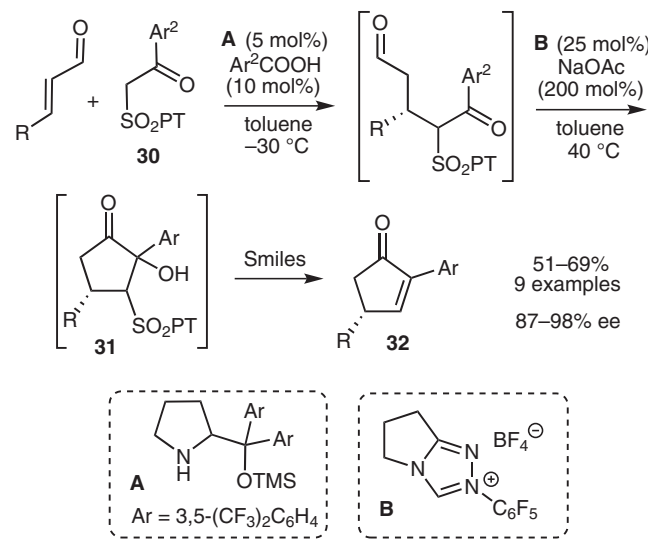

Scheme 10 
Tandem condensation-Wittig cyclization reactions between 2-oxo- or 2,4-dioxo-alkylidinephosphoranes and glyoxals or diacylolefins represent another $(3+2)$ type of assembly leading to cyclopentenones in an efficient manner, although cyclohexenone formation may be a competing process. Some aspects of this methodology were reviewed recently. ${ }^{48}$ In the reaction of phosphoranes 33 with a series of maleic diesters, the 2-cyclopentenone products 34 were obtained in moderate yields but excellent diastereoselectivities (Scheme 11). ${ }^{49}$ One study was carried out using a chiral sulfoxide derivative of 2-oxopropylidine phosphorane 35 , prepared in situ and treated with a series of $(E)$-enediones. ${ }^{50}$ In the presence of a key copper additive, the Michael addition and subsequent intramolecular Wittig reaction proceeded in a highly regioand stereoselective fashion to give the corresponding 3methyl-5-sulfoxy-2-cyclopentenones 36. Conditions were also established for the facile removal of the sulfoxide adjuvant, and the resulting 3,4-disubstituted 2-cyclopentenones 37 were obtained with very high enantiomeric excess (Scheme 11).
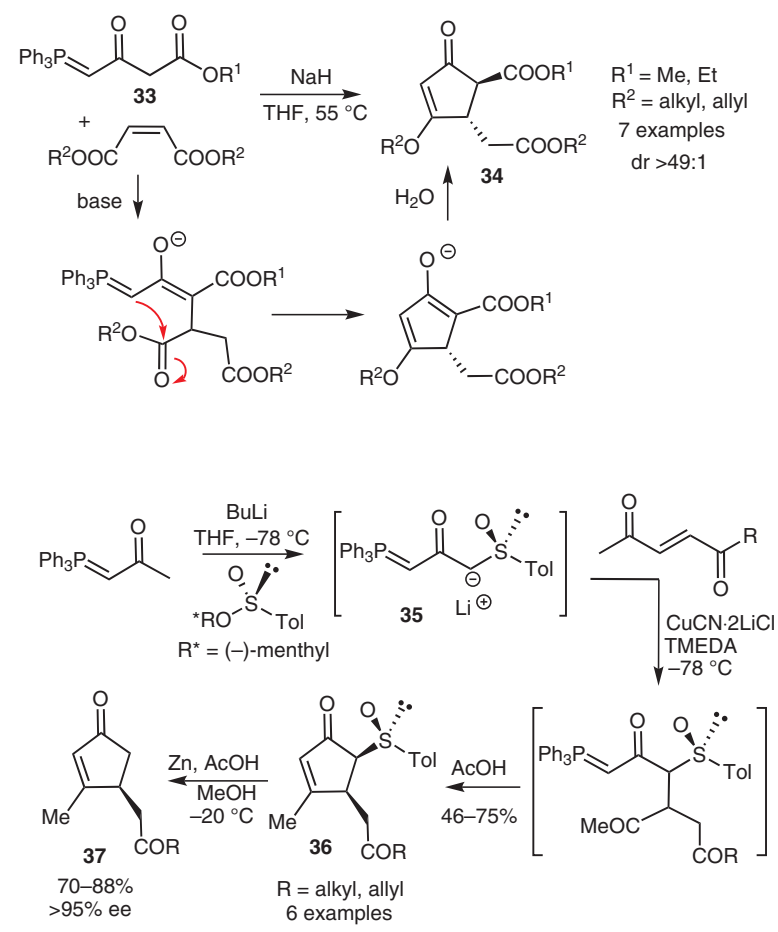

\section{Scheme 11}

Intermolecular condensations involving classical carbanion chemistry appear to offer a simple route to the 2-cyclopentenone core, but side reactions often limit the synthetic utility. Nevertheless, successful applications of such chemistry do appear. A number of recent papers have described crossed-aldol condensations between benzil derivatives and selected ketones to give highly functionalized 4-hydroxy-2-cyclopentenones, usually as diastereoisomeric mixtures. ${ }^{51}$

A comprehensive study of the cyclization of 1,2-diketones with 1,3-dicarbonyl dianions $\mathbf{3 8}$, or with the correspond- ing silyl enol ethers 39 under acidic conditions, revealed this to be a convenient and quite general approach for the preparation of a series of 2-acyl-4-hydroxy-2-cyclopentenones, 40 or 41, respectively (Scheme 12). The basemediated reactions required a silica gel treatment to induce the cyclization, while the more direct silyl enol ether approach gave slightly lower yields. ${ }^{52}$

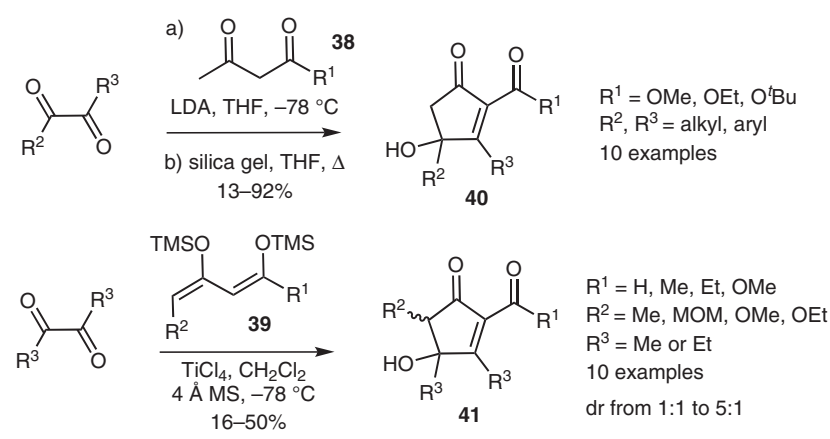

Scheme 12

The reaction of 3-substituted allenoates $\mathbf{4 2}$ with symmetrical diaryl 1,2-diketones in the presence of a phosphine gave highly substituted 2-cyclopentenones $\mathbf{4 3}$ in excellent yields (Scheme 13). The reaction appeared to be highly diastereoselective although data were not given. The reaction also proceeded with unsymmetrical diones, although without regioselectivity. A zwitterionic adduct formed from the allene and the phosphine was proposed as the key reactive intermediate, which first attacked one carbonyl with elimination of water then attacked the second carbonyl with water assistance to induce cyclization. ${ }^{53}$

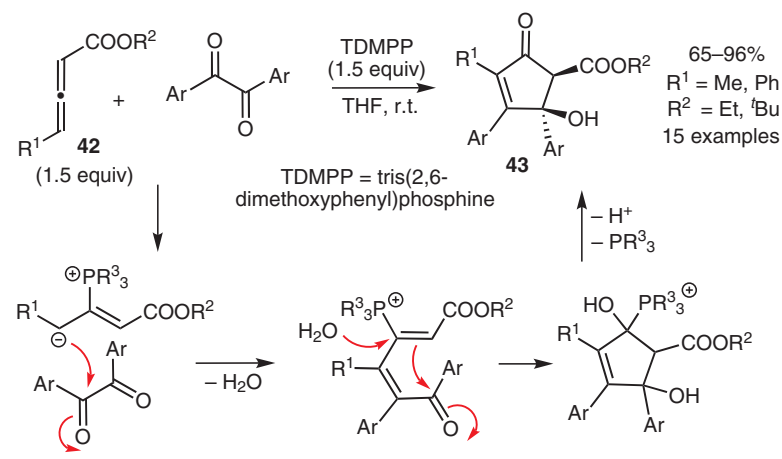

Scheme 13

\section{(4+1) Ring Assembly}

An alternative approach to the 2-cyclopentenone core is a $(4+1)$ assembly, which has the clear advantage of removing regioselectivity issues. Carbon monoxide is the obvious 'one-carbon' component, providing $\mathrm{C} 1$ of the target structure, but a few other reagents have been used successfully to provide $\mathrm{C} 3$ or $\mathrm{C} 5$ when combined with appropriate 'four-carbon' partners.

Carbonylative cyclization of 1,3-butadiene derivatives in the presence of carbon monoxide has been known for some time, and is still considered a pertinent strategy. A 
series of bicyclic enones $\mathbf{4 5}$ was prepared in excellent yields from the appropriate halogeno-dienes 44 using a palladium catalyst under an atmosphere of carbon monoxide (Scheme 14). ${ }^{54}$

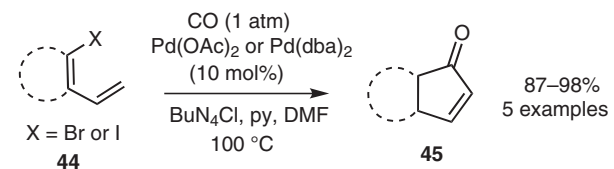

Scheme 14

An interesting recent development is the carbonylation of a 1-lithiobutadiene $\mathbf{4 6}$ followed by spontaneous cyclization to give a cyclopentadienyl enolate 47 (Scheme 15). It was shown that this intermediate could be trapped by acylation at the $\gamma$-position, providing the corresponding 2cyclopentenone 48 in a one-pot process. However, the system was sensitive to steric factors and $\alpha$ - or O-acylation products were obtained when the 4,5-positions were substituted. ${ }^{55}$

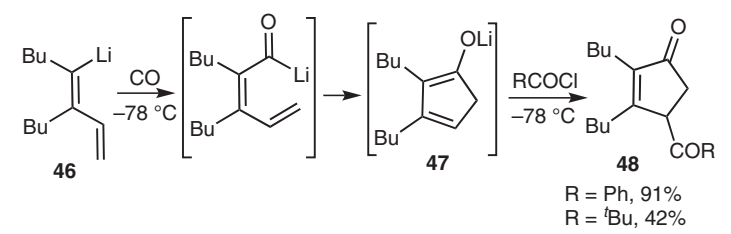

Scheme 15

A titanium-mediated (4+1) assembly of 1,3-butadienes and nitriles has been described, in which the nitrile acts as the 'one-carbon' component. ${ }^{56}$ Treatment of 2-silyloxybutadiene 49 with titanium isopropoxide and a Grignard reagent gave a titanacyclopentene intermediate which reacted with a nitrile to give a silyloxycyclopentenylamine 50; spontaneous hydrolysis during work-up led to the corresponding 2-cyclopentenone 51 directly (Scheme 16).
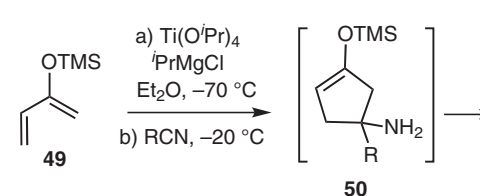

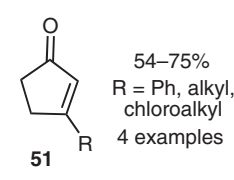

Scheme 16

Vinyl ketenes are useful intermediates in synthesis and they can be stabilized to some extent as trialkylsilyl derivatives. A selection of such vinyl ketenes $\mathbf{5 2}$ reacted with nucleophilic carbenes, generated in situ thermally, to provide highly substituted 2-cyclopentenones $\mathbf{5 3}$ in good yields (Scheme 17). ${ }^{57}$ In another study, the reaction of vinyl ketene 54 with selected $\alpha$-benzotriazolyl (Bt) organolithium reagents 55 gave the 2-cyclopentenones 56 in fair to good yields, although the addition of a Lewis acid was sometimes necessary to facilitate departure of the $\mathrm{Bt}$ group (Scheme 17). The products in this case were ob- tained with good trans-stereoselectivity, particularly when the 5-position was monosubstituted. ${ }^{58}$
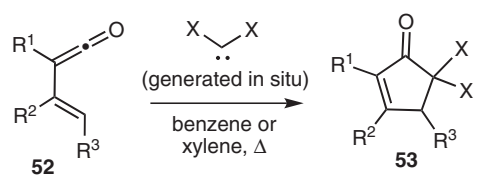

$$
\begin{gathered}
55-88 \% \\
\mathrm{R}^{1}=\mathrm{TMS} \text { or TIPS }^{\mathrm{T}} \mathrm{X}=(\mathrm{OMe})_{2},(\mathrm{SPr})_{2}, \\
-\mathrm{NPhCH}_{2} \mathrm{CH}_{2} \mathrm{NPh}- \\
8 \text { examples }
\end{gathered}
$$<smiles>CC=C(C)C(=O)SCCC</smiles>

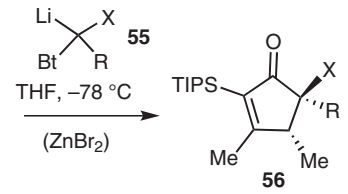

$$
\begin{gathered}
34-85 \% \\
\mathrm{R}=\mathrm{H} \text { or Me } \\
\mathrm{X}=\text { heteroatomic function } \\
9 \text { examples } \\
\text { dr from } 74: 26 \text { to }>99: 1
\end{gathered}
$$

Scheme 17

A selection of stable silyl vinyl ketenes bearing tricarbonylchromium $(0)$ arene substituents 57 were prepared from Fischer carbene complexes and alkynes, and reacted with diazomethane, or a derivative thereof, to give the (4+1)annulation products 58 in excellent yields and in a completely stereoselective fashion (Scheme 18). ${ }^{59}$ Removal of the chromium moiety was subsequently achieved using cerium(IV) ammonium nitrate. This process was applied in an elegant intramolecular mode, to provide an efficient synthesis of the rocaglamide skeleton, whereby the Fischer carbene alkyne $\mathbf{5 9}$ was transformed in a three-step process into adduct $\mathbf{6 0}$ in good yield and complete stereoselectivity (Scheme 18). ${ }^{60}$ Related studies showed that the Köbrich reagent $\left(\mathrm{CH}_{2} \mathrm{I}_{2}\right.$ with BuLi) could be used instead of a diazoalkane, while the use of tert-butyl isocyanate provided the 2-cyclopentenone core with an exocyclic $(Z)$-imine moiety at the 5-position. ${ }^{61}$

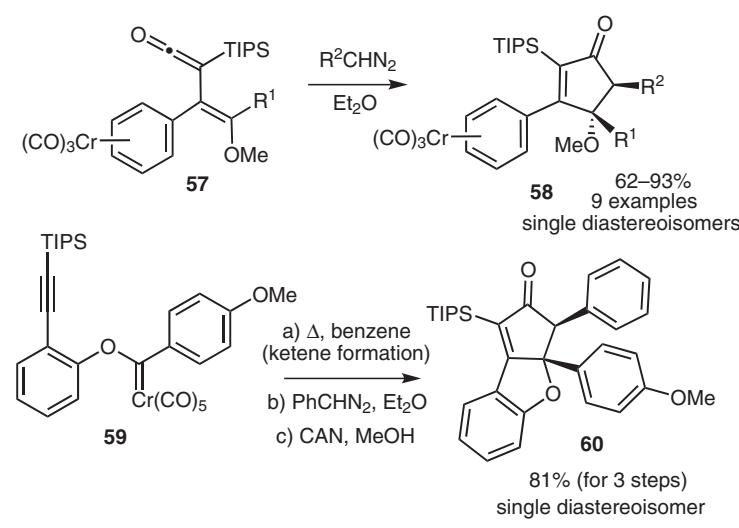

Scheme 18

The reaction of methylenecyclopropanes $\mathbf{6 1}$ with Fischer carbene chromium complexes $\mathbf{6 2}$ provided 2-cyclopentenones $\mathbf{6 3}$ in which the ring had been formed from all four of the methylenecyclopropane carbon atoms plus one equivalent of carbon monoxide (Scheme 19). The proposed mechanism involved an initial $[2+2]$-cycloaddition followed by a rearrangement to give an intermediate alkylidinemetallacyclopentane which then underwent $\mathrm{CO}$ insertion, chromium elimination, and finally isomerization. ${ }^{62}$ 


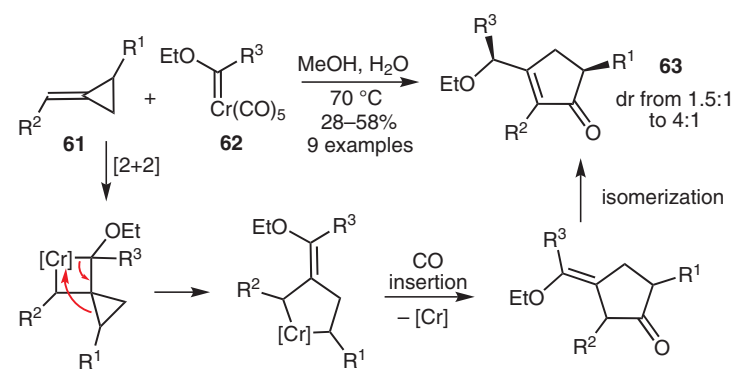

Scheme 19

\section{$3 \quad$ Cyclizations}

In this review, 'cyclization' implies the formation of a new ring structure from an acyclic molecule (or from an acyclic fragment of a larger molecule) through the formation of one new $\sigma$ bond. This definition includes cases where a new $\pi$ system is generated (or an existing $\pi$ system is shifted) as the $\sigma$ bond is formed. A comprehensive review of ring-closure approaches to cyclopentane derivatives, including some useful precursors of 2-cyclopentenones, appeared recently. ${ }^{63}$

\section{Nazarov Cyclization}

Arguably one of the most important methods for the preparation of 2-cyclopentenones is the acid-promoted cationic pericyclization of a divinyl ketone, first reported by Nazarov in $1944 .{ }^{64}$ It is now established that the reaction is initiated by acid complexation of the ketone to give a pentadienyl cation which undergoes a $4 \pi$-electron cyclization in a conrotatory fashion to provide an oxyallyl cation intermediate. Elimination of a proton followed by reprotonation of the acid-bound enolate gives the 2-cyclopentenone product (Scheme 20).

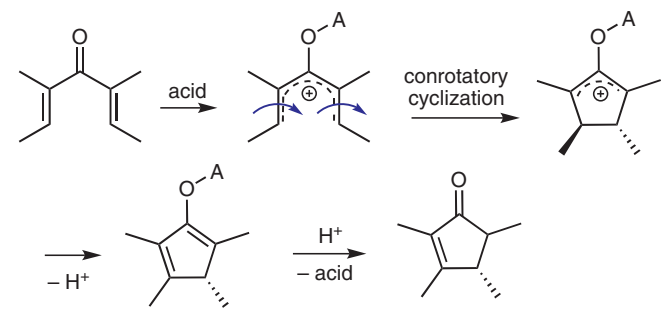

\section{Scheme 20}

The obvious synthetic potential was for some time offset by selectivity issues, involving the regiochemistry of the proton elimination step and the stereochemistry of the enolate protonation step, as well as the harsh acidic conditions which were often required. As work progressed, it emerged that steric and/or electronic effects (particularly polarized double bonds) could be harnessed to control the selectivity, and milder reaction conditions were discovered. Significant recent developments include the use of organocatalysts $^{65}$ and transition-metal-complex catalysts, ${ }^{66}$ which open the way to enantioselective reactions and/or tandem transformation sequences. The Nazarov reaction has established itself in the modern synthetic chemist's toolbox, and progress has been documented regularly in comprehensive reviews, particularly in the last decade, ${ }^{67}$ and include focuses on catalytic versions, ${ }^{68}$ asymmetric versions, ${ }^{69}$ and alternative routes to the intermediate pentadienyl cation in order to circumvent the highly reactive divinyl ketone substrates. ${ }^{70}$ Processes in which the cyclopentenyl cation intermediate is intercepted by a nucleophile constitute a rich and developing area referred to as 'interrupted Nazarov reactions', but generally they deviate the reaction course away from 2-cyclopentenone formation. ${ }^{71}$

Only a few of the many recent elegant applications of the Nazarov cyclization are presented here (Scheme 21). In a synthesis of $( \pm)$-xanthocidin, very fast cyclization of the highly substituted divinyl ketone 64 was achieved using iron(III) chloride. The sterically challenged oxyallyl cation intermediate underwent exocyclic elimination leading to the 5-methylene-2-cyclopentenone product $\mathbf{6 5}$ in less than three minutes. ${ }^{72}$ The preparation of a 2-hydroxycyclopentenone core can be achieved using a vinyl diketone as the precursor. As part of the total synthesis of (+)-fusicoauritone, the acidic treatment of the macrobicycle $\mathbf{6 6}$ with a Lewis acid gave the requisite tricyclic product $\mathbf{6 7}$ with an all-syn stereochemistry. ${ }^{73}$ The Nazarov cyclization of the 3-acylated benzofuran $\mathbf{6 8}$ was the key step in a short formal synthesis of $( \pm)$-methyl rocaglate; while other Lewis acids only induced a retro-Friedel-Crafts reaction, acetyl bromide was able to induce cyclization to give 69 in very good yield. ${ }^{74}$ A chiral Brønsted acid was used to catalyze the cyclization of $\mathbf{7 0}$ with excellent yield and torquoselectivity, to furnish 71 with $82 \%$ ee in a concise formal synthesis of $(+)$-roseophilin. In principle, water is required to trap the oxyallyl intermediate and presumably was furnished by the reagent-grade carbon tetrachloride used as the solvent. ${ }^{75}$ Copper(II)-complex-mediated cyclizations may be accompanied by skeletal rearrangements, again at the oxyallyl cation stage, and such a process was exploited in a total synthesis of enokipodin B. A bulky bisoxazolidine copper(II) complex induced a sequential cyclization-double-[1,2]-Wagner-Meerwein shift transformation of divinyl ketone $\mathbf{7 2}$ (as an easily isomerized mixture, of which only the $Z$-isomer reacted) to give the 2-cyclopentenone $\mathbf{7 3}$ with impressive regioselectivity, although the enantioselectivity was poor. ${ }^{76}$ 


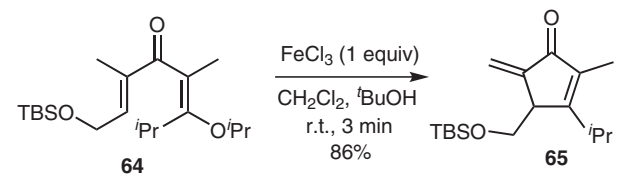

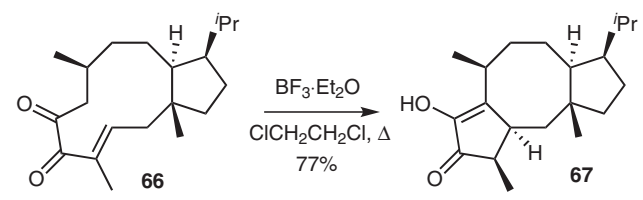
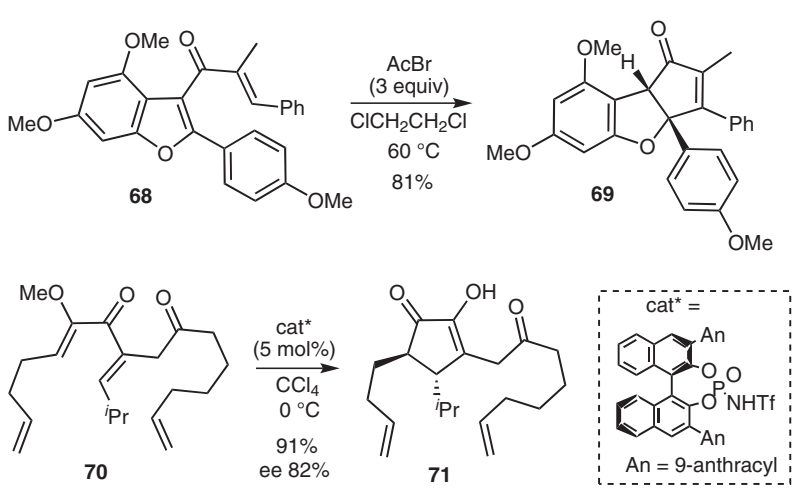<smiles>COc1cc(C=C(C(=O)C(C)=C(C)C)C(=O)C(C)C)ccc1C</smiles>

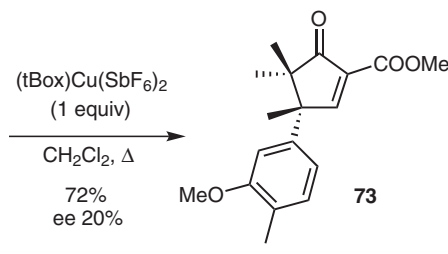

Scheme 21

Treatment of chiral oxazolidinone-derived divinyl ketones $\mathbf{7 4}$ with methanesulfonic acid gave good yields of Nazarov cyclization products $\mathbf{7 5}$ as single regioisomers and with excellent diastereoselectivity (Scheme 22). ${ }^{77} \mathrm{~A}$ unique role for the chiral auxiliary was proposed during the cyclization process, whereby two transition-state conformers are involved, each of which progresses with the same torquoselectivity directed by allylic strain. ${ }^{78}$
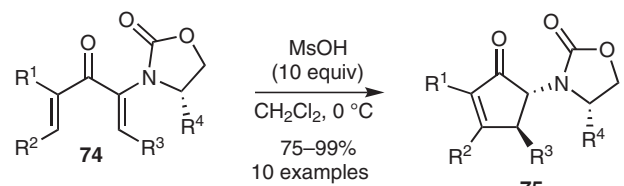

$R^{1}, R^{2}, R^{3}=$ alkyl, aryl 10 examples 75
$d r>20: 1$<smiles></smiles><smiles>[R]Cc1c([2H])c([R])c(O)c(N2C(=O)OC[C@H]2[Z7])c1CP</smiles>

Scheme 22

An impressive multi-step one-pot Wittig-Nazarov protocol was conceived for the construction of 4-alkylidene-2cyclopentenones starting from $\alpha$-diazoketones $\mathbf{7 6}$ and acid chlorides 77 (Scheme 23). The transformation involved iron-catalyzed formation of the stabilized ylide $\mathbf{7 8}$ on the one hand and base-induced formation of a ketene on the other hand. These intermediates reacted together to form a vinyl allenyl ketene 79, which then underwent trifluoroacetic acid mediated Nazarov cyclization to give the requisite products $\mathbf{8 0}$ in good yields and with a high selectivity for the $Z$-isomer. ${ }^{79}$

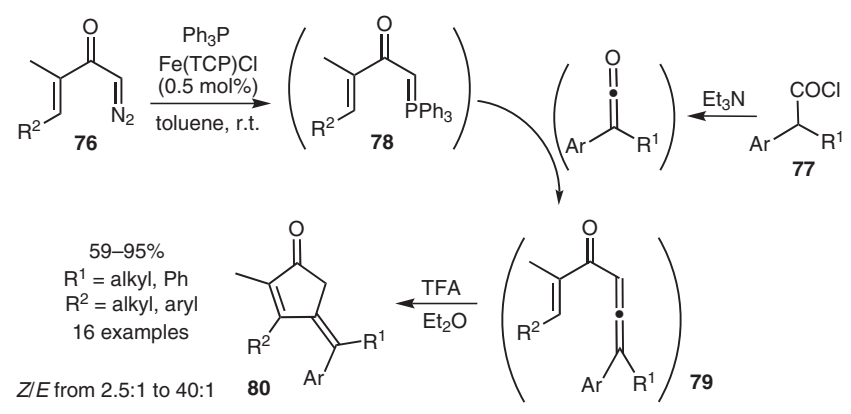

Scheme 23

Substrates other than divinyl ketones have been developed for the generation of pentadienyl cations, which further enhances the scope of conrotatory $4 \pi$-electron cyclizations. Reactions in this category are commonly referred to as Nazarov cyclizations, although there is some convergence with the Rautenstrauch rearrangement and perhaps also with pentadienal cyclizations (vide infra). Reduction of vinylalkylidine dioxolanones $\mathbf{8 1}$ provided the corresponding 5-hydroxy-2-cyclopentenones $\mathbf{8 2}$ as single diastereoisomers, presumably via conrotatory closure of an intermediate 1,2-oxidopentadienyl cation (Scheme 24) ${ }^{80}$ The strategy was therefore applied in a key transannulation step in a total synthesis of $( \pm)$-cephalotaxine. ${ }^{80}$ Selective oxidation of alkoxyallenes 83 using dimethyldioxirane provided an entry to oxypentadienyl zwitterions, which cyclized in a Nazarov fashion to give the bicyclic adducts $\mathbf{8 4}$, often as single cis-isomers (Scheme 24). A mechanism involving diastereoselective epoxidation directed by the difference in the steric bulk of the allene substituents followed by the usual concerted $4 \pi$ electron cyclization was evoked to explain the diastereoselectivity. ${ }^{81}$
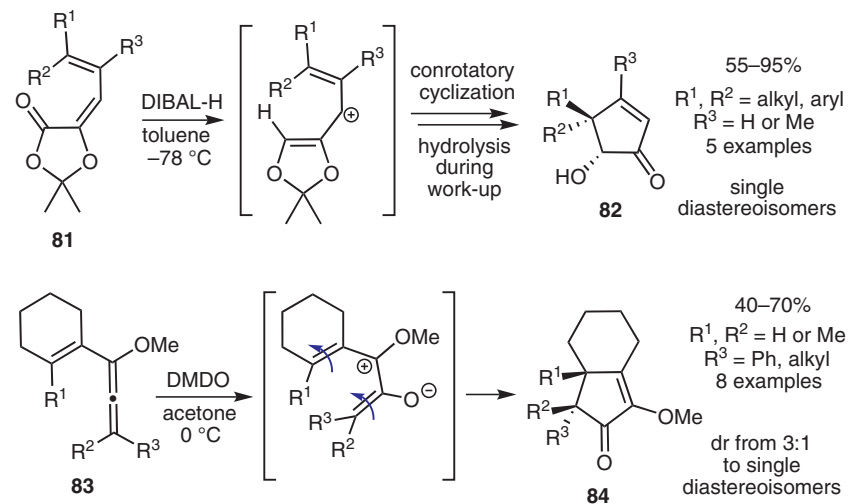

Scheme 24 


\section{Rautenstrauch Rearrangement}

The palladium(II)-catalyzed isomerization of 1-ethynyl2-alkenyl acetates to give 2,3-disubstituted 2-cyclopentenones, was first reported in $1984 .{ }^{82}$ The proposed mechanism involves consecutive metal additions to the $\pi$ systems and migration of acetate, with a hydrolysis step to liberate the enone (Scheme 25).

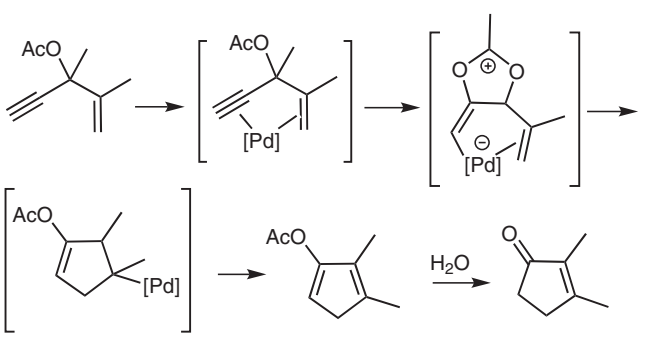

Scheme 25

The palladium(II) version of the reaction is still employed today: in a stereoselective assembly of the $\mathrm{ABCE}$ ring system of the natural product azadirachin, the $\mathrm{E}$ ring was constructed in this way from the enyne ester $\mathbf{8 5}$, giving the tetracyclic adduct 86 as a 1:1 mixture of diastereoisomers (Scheme 26). ${ }^{83}$
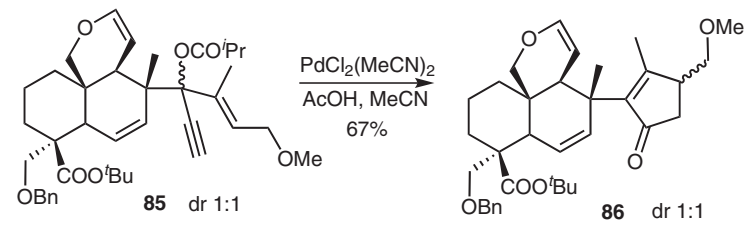

Scheme 26

Most recent developments of this reaction have focused on the use of gold(I) catalysts. A plausible mechanism involves initial formation of an alkyne-gold complex which undergoes a [1,2]-shift of carboxylate to generate a goldcoordinated allylic cation, which then evolves by a Nazarov-type cyclization process. The resulting acyloxy cyclopentadiene is hydrolyzed to give the bicyclic product. The standard process was applied to a series of 1ethynyl-2-alkenyl pivaloates $\mathbf{8 7}$ to allow access to a wide range of 3,4- or 3,5-disubstituted 2-cyclopentenones $\mathbf{8 8}$ under mild conditions (Scheme 27) ${ }^{84}$ When a nonracemic substrate 89 was used, conditions were found in which the 3,4-disubstituted products 90 could be obtained with excellent chirality transfer. ${ }^{84}$ In related work, 1-ethynyl-1allenylalkyl acetates 91 underwent cycloisomerization to acetoxyfulvenes 92, in the presence of a cationic bisoxazolidine-gold(III) complex, that then evolved to provide 4-methylene-2-cyclopentenones $\mathbf{9 3}$ upon methanolysis. ${ }^{85}$ In further developments of this theme, conjugated enynyl derivatives have been cyclized, again with gold-mediated migration of an oxygen function (Scheme 28). Enynyl acetates 94 were treated with a gold(I) complex to give
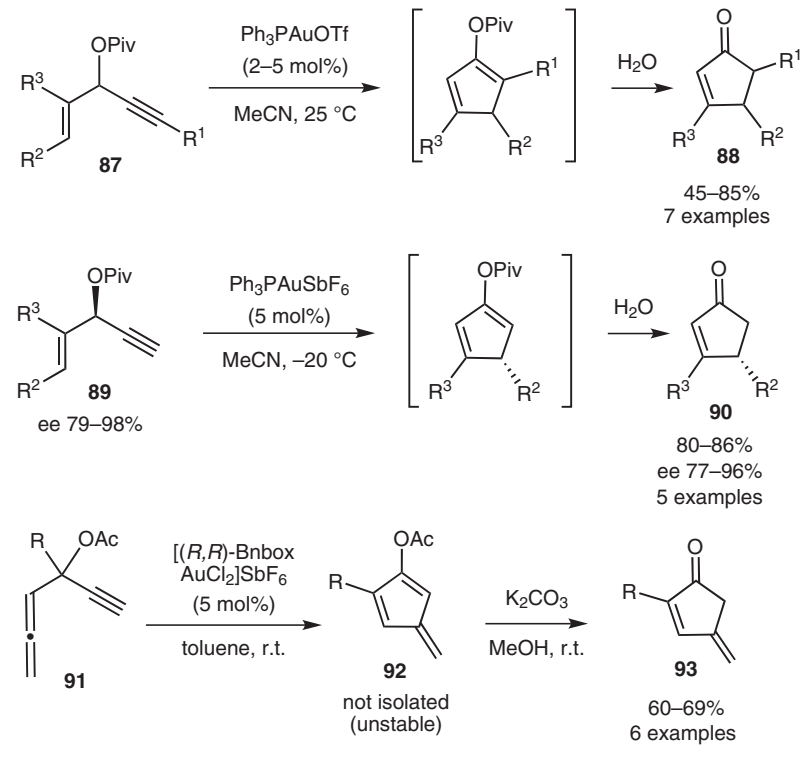

Scheme 27

3,5-disubstituted or 3,4-fused bicyclic 2-cyclopentenones 95. It was suggested that tandem gold(I)-catalyzed [3,3]rearrangement of the substrate and activation of the allenic acetate led to the gold-coordinated allylic cation intermediate, which cyclized as indicated above. ${ }^{86}$ 5-Silyloxypent-3-en-1-ynes 96 underwent cyclization when treated with a gold(I) catalyst, to give 2-cyclopentenones 97 in generally good yields. In this case it was proposed that gold complexation of the alkyne induced siloxycyclization followed by carbon-oxygen bond fragmentation to give an allylic carbocation, which subsequently cyclized at the gold-bound alkenyl site, leading to the substituent topology observed in the final products. ${ }^{87}$

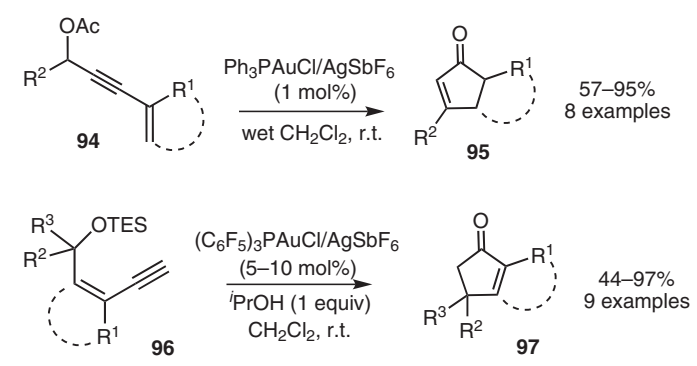

Scheme 28

The hydrative rearrangement of 1,1-diethynylcarbinol acetates 98 to 5-acetoxy-2-alkyl-2-cyclopentenones 99 was achieved using a gold(I) catalyst (Scheme 29). The substrates first underwent a gold-mediated [3,3]-rearrangement to allenyne acetates which then reacted further by gold-induced oxacyclization then nucleophilic attack by water. Gold-promoted 5-endo-dig cyclization provided the cyclic products, accompanied by small amounts of the allenone by-product 100. ${ }^{88}$ 


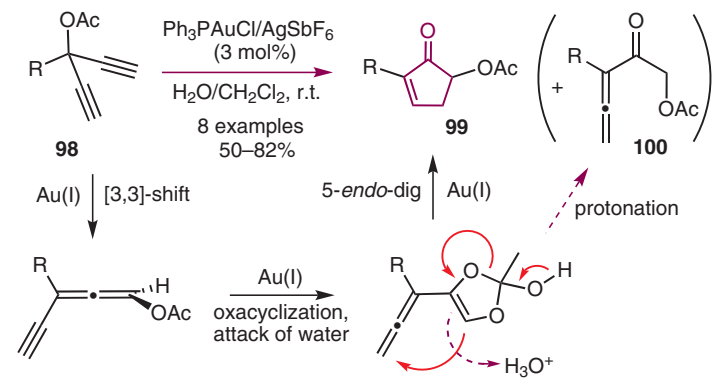

Scheme 29

\section{Hydrative Carbocyclization}

In a somewhat different fashion from the reactions described above, the hydrative carbocyclization of 1,5-diyn3-ones 101 was achieved using a gold(I) catalyst to furnish 4-acyl-2-cyclopentenones 102, although other acyclic products were obtained as well (Scheme 30). An important difference compared to the Rautenstrauch process is the absence of oxygen moiety migration, meaning that the ketone carbon of the substrate was retained as $\mathrm{Cl}$ in the cyclic product. One of the alkyne carbon atoms was incorporated as the acyl function in an exocyclic locus in the product structures. It was suggested that the goldmediated hydration of the electron-rich alkyne generated a gold enolate which then cyclized to a gold cyclopentenonyl intermediate. ${ }^{89}$

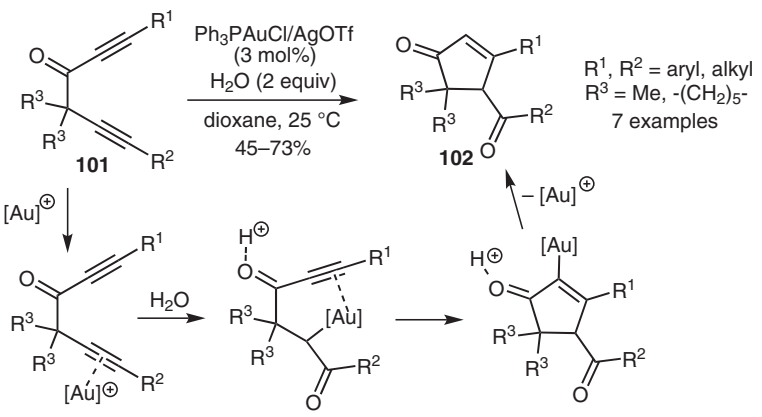

Scheme 30

\section{Ring-Closing Metathesis}

One of the most important developments in metathesis has been to provide a tool for the closure of organic ring systems. Ring-closing metathesis reactions have been reviewed regularly, notably with regard to their applications to natural product synthesis. ${ }^{90}$ Five-membered-ring closure is generally easy, but the reaction is sensitive to electronic factors, with electron-poor alkenes being lessfavored substrates. As a result, most ring-closing metathesis studies have targeted 3-cyclopentenols, although these compounds are often readily oxidized to 2-cyclopentenones without difficulty. Here, we report only on ring-closing metathesis reactions that provide 2-cyclopentenones directly.

Despite the involvement of a deactivated alkene, the Grubbs I catalyst induced the cyclization of diene $\mathbf{1 0 3}$ to provide 2-cyclopentenone 104 in 67\% yield (Scheme 31). ${ }^{91}$ The use of the Grubbs II catalyst to transform the substrates $\mathbf{1 0 5}$ was more efficient, and provided the series of 2- and/or 4-substituted 2-cyclopentenones 106 in excellent yields. ${ }^{92}$ As part of the total synthesis of (-)-heptemerone $\mathrm{B}$, the ring-closing metathesis reaction of the highly substituted substrate $\mathbf{1 0 7}$ was conducted using Grubbs II without incident to give the key intermediate 108, again in excellent yield. ${ }^{93}$ Comparable ring-closing metathesis processes were used to obtain single enantiomers of Bocprotected 2- and 5-amino-2-cyclopentenones. ${ }^{94}$

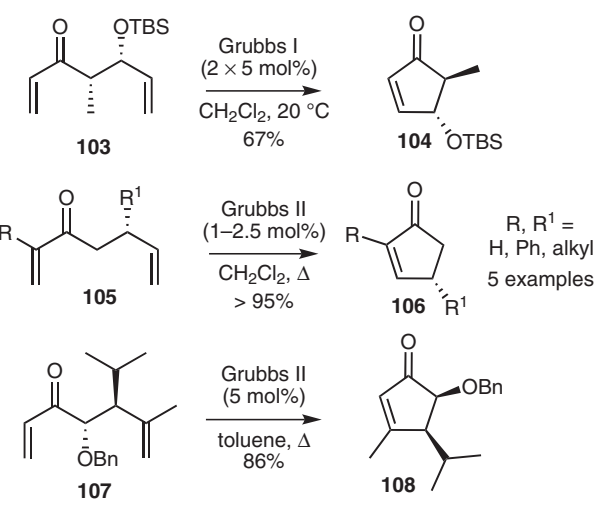

Scheme 31

Tandem ring-opening and ring-closing procedures have been described within the context of elegant syntheses of complex natural product skeletons (Scheme 32). For the construction of the tricyclic core of tricycloclavulone, readily available compound $\mathbf{1 0 9}$ was treated with the Grubbs I catalyst to provide the requisite bicyclo[3.3.0] ring system of 110 in good yield. ${ }^{95}$ A tandem ring-opening and double-ring-closing metathesis protocol was applied in a total synthesis of $(+)$-cyanthiwigin U. Starting from the dialdehyde 111, double vinyl Grignard addition followed by oxidation gave the alkene-bis(enone) 112 which was treated immediately with the Grubbs II catalyst to provide the desired tricyclic product 113 in $43 \%$ yield for three steps. ${ }^{96}$
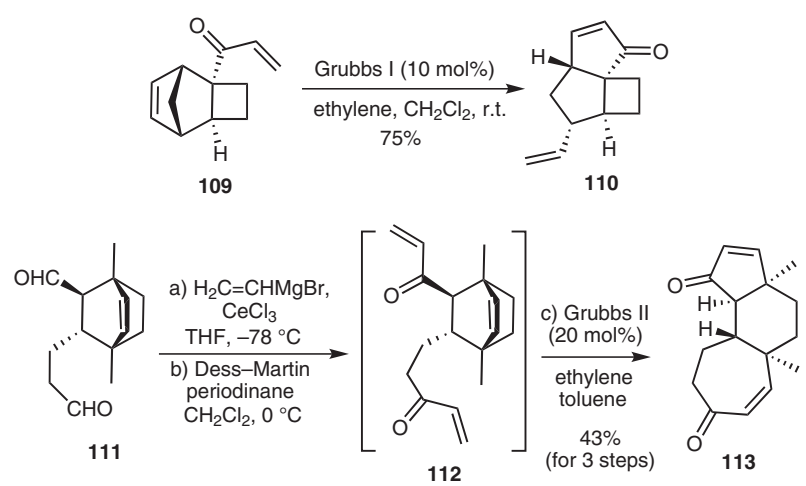

Scheme 32

The metathesis reactivity of 2-alkylidene-1,3-dicarbonyls is low; even so, when substrate $\mathbf{1 1 4}$ was subjected to the 
Grubbs II catalyst, the highly substituted cyclic keto ester 115 was obtained in an acceptable 65\% yield (Scheme 33). With the less bulky substrate 116, the requisite ringclosing metathesis product $\mathbf{1 1 7}$ was obtained in higher yield using a lower catalyst loading. ${ }^{97}$

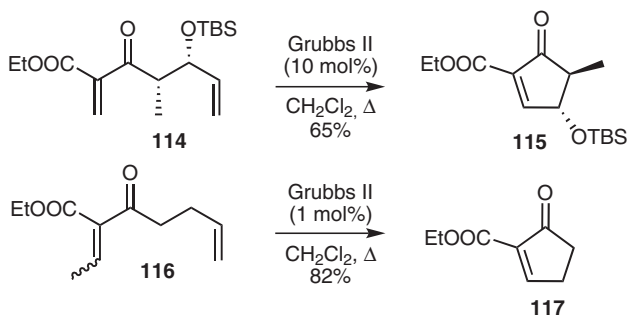

Scheme 33

Related to the ring-closing metathesis reaction is the socalled ring-closing enyne metathesis, which can provide a useful access to vinylcyclopentenes. ${ }^{98}$ However, the reaction is not efficient with the appropriate precursors for the formation of 2-cyclopentenones (Scheme 34). Cyclization of the alkene-ynone 118 in the presence of Grubbs II catalyst gave the 2-vinyl-2-cyclopentenone 119 in $32 \%$ yield, ${ }^{91}$ while the alternative alkyl-enone mode prevalent in 120 (albeit with a deactivated enone) evolved only in the presence of titanium(IV) isopropoxide and even then gave a meager $4 \%$ yield of the 3-vinyl-2-cyclopentenone product 121, accompanied by other cross-metathesis products. $^{97}$
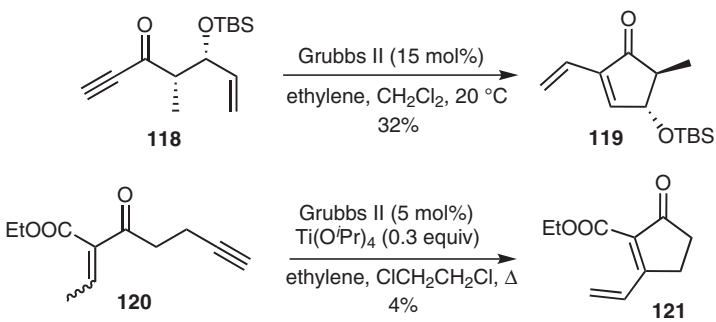

Scheme 34

\section{Other Transition-Metal-Mediated Cyclizations}

Intramolecular oxidative Heck coupling has been reported using vinyl 2-bromovinyl carbinols $\mathbf{1 2 2}$ as substrates: the 5-endo-trig cyclization gave the 2-cyclopentenones $\mathbf{1 2 3}$ directly in decent yields (Scheme 35). ${ }^{99}$ With 2-methylallyl 2-bromovinyl carbinol substrates 124, the cyclization mode switches to 5-exo-trig which furnishes the corresponding 4,4-dimethyl-2-cyclopentenones 125 in good yields. ${ }^{100}$ Propargyl 2-bromovinyl carbinols $\mathbf{1 2 6}$ have also been transformed into 4-methyl-2-cyclopentenones 127, although in this case the yields were modest. ${ }^{101}$

The Liebeskind-Srogl coupling reaction was applied in intramolecular mode to a highly functionalized 2-vinylstannane thioester 128 (Scheme 36). After optimization,

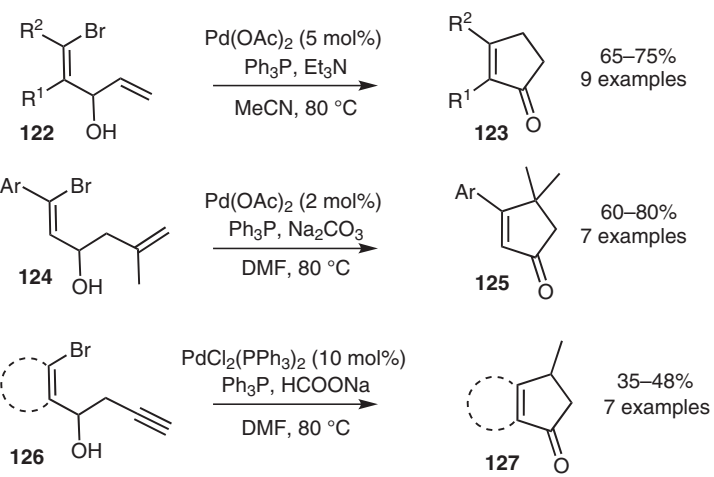

Scheme 35

the desired 2-cyclopentenone 129 was obtained in high yield, opening the way for an expedient total synthesis of litseaverticillols A and B. ${ }^{102}$

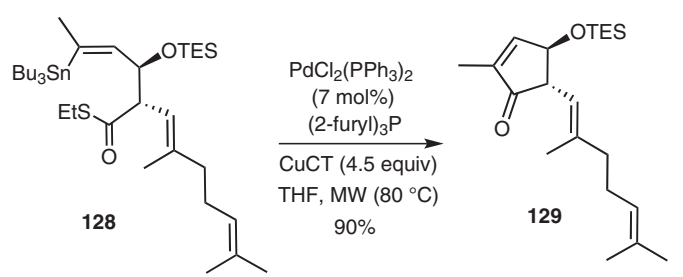

Scheme 36

4-Alkynals 130 were converted into 2-cyclopentenones 131 by way of a rhodium(I)-catalyzed intramolecular hydroacylation process (Scheme 37). Substituents in any position were compatible with the process, although no 4,5disubstituted case was examined; acetone was required as the solvent in order to obtain good yields. ${ }^{103}$ With a coordinating methoxy group in the 4-position of the substrates 132, the employment of a chiral phosphine ligand and a noncoordinating solvent, the hydroacylation provided an excellent kinetic resolution, giving the enantiomerically enriched 4-methoxy-2-cyclopentenones 133. The other enantiomers of the substrates $\mathbf{1 3 2}$ were either recovered intact, or were converted into the isomeric 2-alkylidenecyclobutanones 134, depending on the phosphine ligand employed. ${ }^{104}$
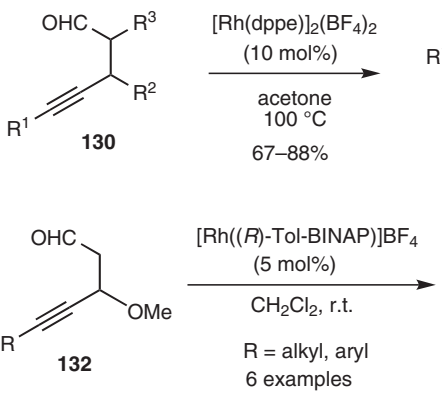

Scheme 37 


\section{Pentadienal Cyclizations}

The $\delta$-carbon of a doubly conjugated carbonyl moiety is not particularly nucleophilic, and few attempts to effect cyclization of such compounds had been described until recently. A study of the reactivity of simple 2,4-dienals 135 in the presence of a Lewis acid demonstrated the feasibility of the approach but also revealed some limitations: the 2-cyclopentenones $\mathbf{1 3 6}$ were obtained in only modest yields (Scheme 38). ${ }^{105}$ The cyclic aliphatic 2,4-dienal 137 provided the corresponding bicyclic enone 138 in poorer yield, and the cyclization failed entirely when the $\gamma$-methyl group was absent, or when attempted with a triple-conjugated substrate. Several mechanistic possibilities were suggested, implicating the formation of a cyclopentadiene epoxide either by a concerted process or via a Nazarovlike mechanism involving the conrotatory $4 \pi$-electron cyclization of an oxypentadienyl cation; isomerization of the epoxide in the acidic medium would account for the formation of the final products (Scheme 38). ${ }^{105}$

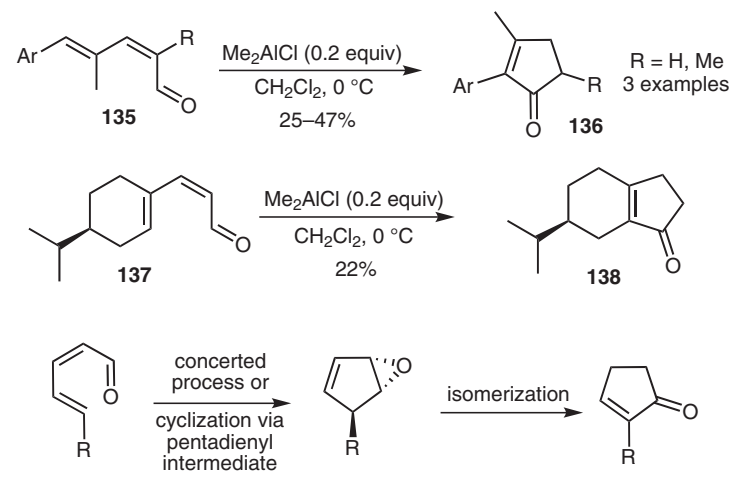

Scheme 38

An improvement was devised, on the premise that the $\delta$ carbon nucleophilicity would be enhanced by making it a part of a vinylogous allyl silane system. In the event, when the silylated precursors 139 were treated with a Lewis acid they provided, after isomerization, the spiro derivatives $\mathbf{1 4 0}$ as single diastereoisomers in good yields (Scheme 39). The excellent diastereoselectivity was explained by a preferred carbonyl coordination by the Lewis acid from the less-hindered face of the six-membered ring. ${ }^{106}$ In a study of the various cyclization modes possible for the cyclic dienal 141, it was found that platinum(II) chloride in the presence of $p$-toluenesulfonic acid, the latter being used to induce isomerization, drove the reaction to exclusive formation of the fused 2-cyclopentenone 142. ${ }^{107}$

\section{Base-Induced Annulations}

Base-mediated condensations of carbonyl compounds have been a mainstay of organic synthesis for over a century. They are still popular methodologies, notably for the construction of cyclic structures, including the title family of compounds. A few recent applications are presented here.

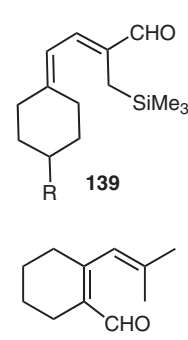

141

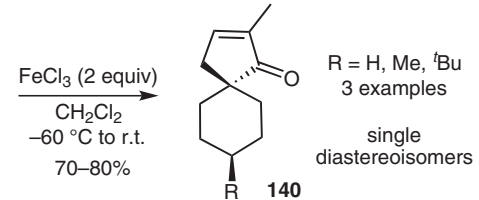

$\mathrm{PtCl}_{2}(5 \mathrm{~mol} \%)$ $\mathrm{TsOH}(5 \mathrm{~mol} \%)$ toluene, $100{ }^{\circ} \mathrm{C}$ $88 \%$<smiles>CC1(C)CC2=C(CCCC2)C1=O</smiles>

Scheme 39

Intramolecular aldolization of a 1,4-diketone or a 4-ketoaldehyde moiety followed by crotonization leads conveniently to the 2-cyclopentenone core, usually as the thermodynamically preferred structural isomer (Scheme 40). A series of 4,4-spiroannulated 2-cyclopentenones 144, inspired in part by the acorone natural product skeleton, were prepared from the cyclic keto aldehyde precursors 143 in uniformly good yields regardless of the existing ring size. ${ }^{108}$ The final step in the synthesis of a model azatriquinane $\mathbf{1 4 6}$ was achieved easily by subjecting compound $\mathbf{1 4 5}$ to mild basic conditions. ${ }^{109}$ Sodium hydride was used to cyclize the diketone 147 to provide bicyclic derivative $\mathbf{1 4 8}$ in good yield, as part of the first total synthesis of $(+)$-minwanenone. ${ }^{110}$ The Schöllkopf bislactim 149 was used to prepare the unusual $\alpha$-amino acid 150, featuring the 5,5-disubstituted 2-cyclopentenone moiety, in enantiomerically enriched form. ${ }^{11}$
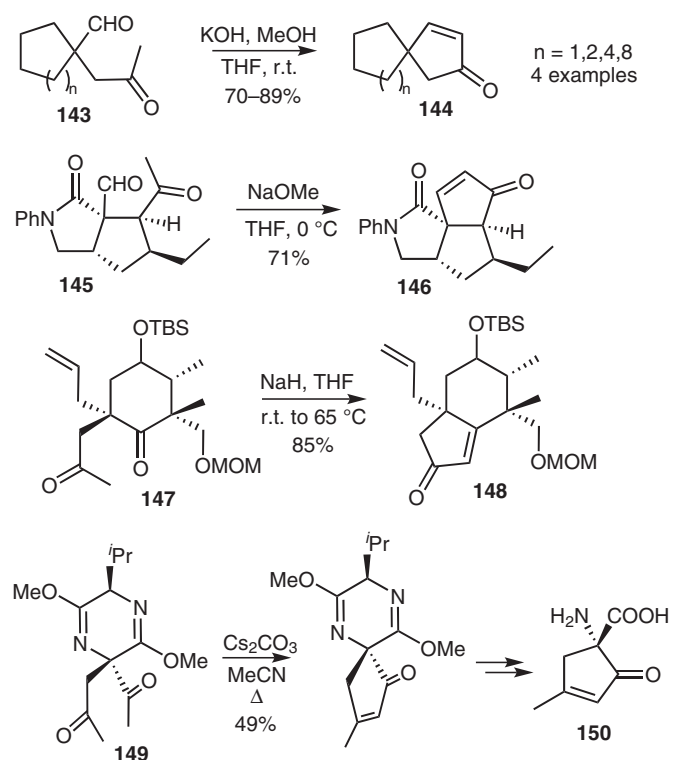

Scheme 40

A thiol-mediated tandem Michael-aldol reaction of the dihydronaphthalene derivatives $\mathbf{1 5 1}$ has been described as a route to fused cyclopentenones $\mathbf{1 5 2}$ (Scheme 41). The proposed mechanism resembles an intramolecular BaylisHillman reaction: the thiol adds in a conjugate manner to the $\alpha, \beta$-unsaturated ester chain of the bicyclic system, 
then base-induced condensation of the $\alpha$-carbanion on the aldehyde, followed by elimination of the nucleophile and prototropy, leads to the 2-cyclopentenone moiety. ${ }^{112}$

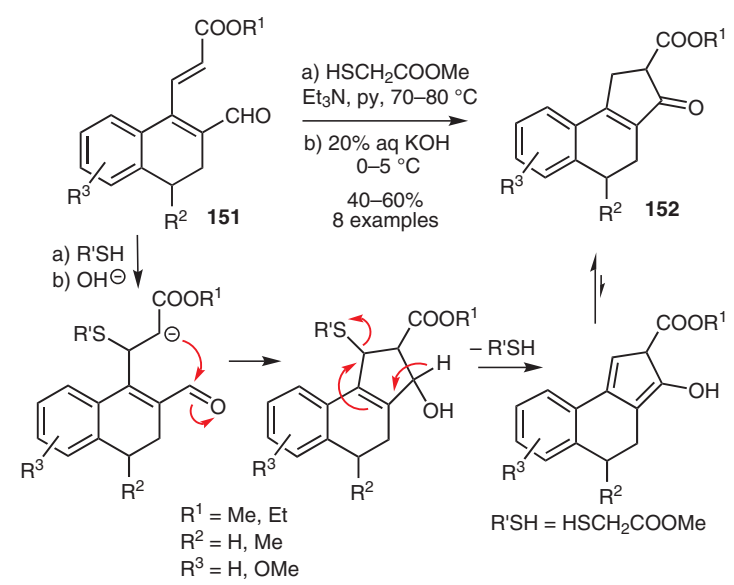

Scheme 41

Other related classical enolate-type condensation procedures have been used fruitfully in intramolecular mode (Scheme 42). With the diketo ester substrate 153, the Knoevenagel reaction was employed to close the fivemembered ring in the 5,7,5-tricyclic system of $\mathbf{1 5 4}$ in a total synthesis of $( \pm)$-sordaricin. ${ }^{113}$ A Knoevenagel reaction was also carried out on the polyfunctional hydroxypyranones 155, which are masked 4-keto-2-enals. Despite a number of potential condensation paths being available, treatment of these compounds with piperidinium acetate gave good yields of the cyclopenta $[b]$ pyran derivatives 156, which were subsequently used to prepare natural product analogues for cytotoxicity evaluation. ${ }^{114}$ The triester 157 was converted smoothly into the cyclopent-2enone-5-carboxylic ester derivative 158 by a Dieckmann cyclization during the initial steps of a short synthesis of (-)-kjellmanianone. ${ }^{115}$

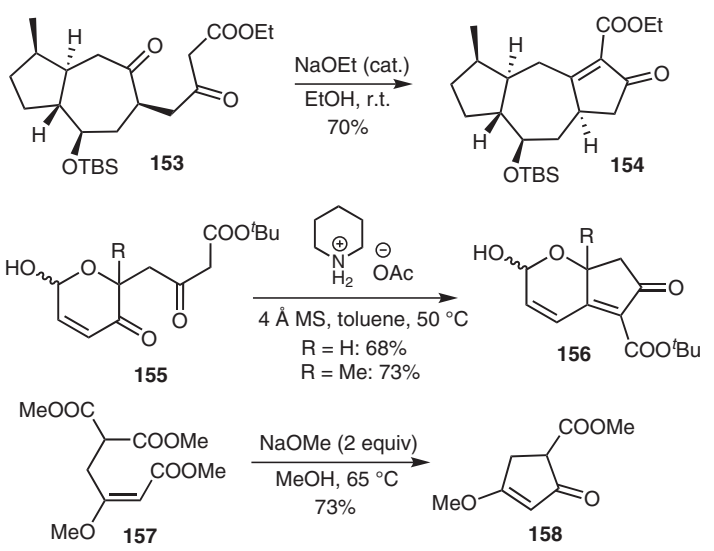

Scheme 42

A novel endocyclic keto-enamine annulation was discovered during a total synthesis of $( \pm)$-cephalotaxine (Scheme 43). Treatment of compound 159 with ferrous sulfate under aerobic conditions provided the pentacyclic adduct 160 in reasonable yield. ${ }^{116}$ The oxygen-dependent acid-mediated formation of a conjugated iminium ion was proposed to account for this reaction.

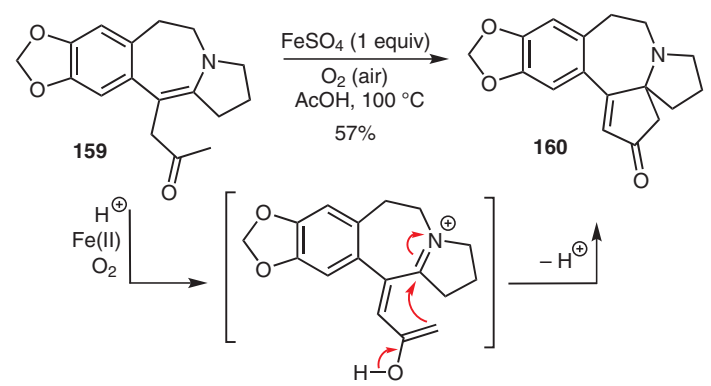

Scheme 43

Using the highly functionalized cyclohexanone 161, an intramolecular Horner-Wadsworth-Emmons reaction was performed to construct the five-membered ring of $\mathbf{1 6 2}$ in good yield during a total synthesis of the neurotrophic modulator $\left( \pm\right.$ )-jiadifenin (Scheme 44). ${ }^{117}$ The detailed study of a range of 2,5-diketophosphonates 163 revealed that the choice of base was critical for the success of the Horner-Wadsworth-Emmons reaction to provide 164. Furthermore, the by-product 165 resulting from a competing intramolecular aldol reaction was sometimes observed, and non-racemic substrates suffered from stereochemical erosion in some cases. ${ }^{118}$
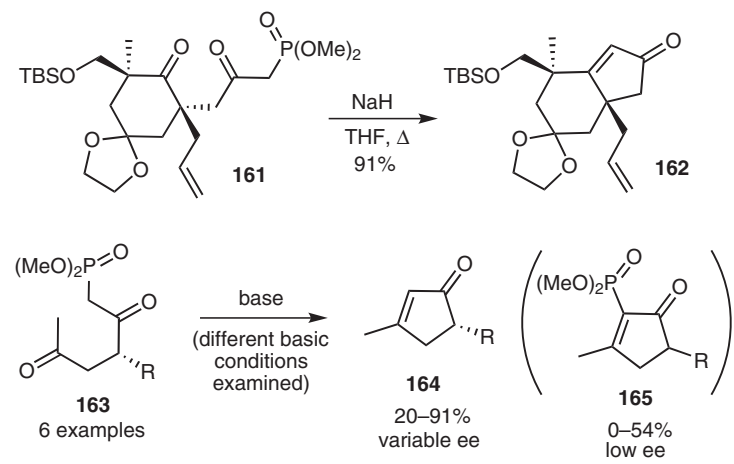

Scheme 44

\section{Other Cyclizations}

Selected $\alpha$-(2-chloro-2-propenyl)- $\alpha$-aryl acetic esters 166 were cyclized under Friedel-Crafts acylation conditions to give the corresponding 3-chloro-5-aryl-2-cyclopentenones 167 (Scheme 45). This was the key step in the development of a short and general method for obtaining 3,5diaryl-2-cyclopentenones $\mathbf{1 6 8} .^{119}$

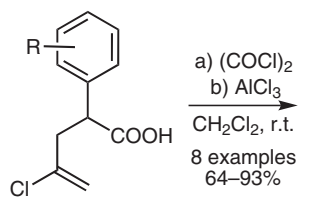

166

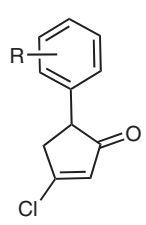

167

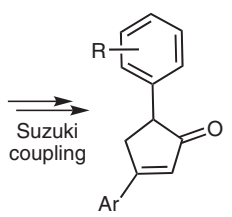

168
Scheme 45 
The rhodium(II)-mediated decomposition of diazo compounds 169 induced an intramolecular $\mathrm{C}-\mathrm{H}$ insertion, to furnish the corresponding cyclopent-2-enone-5-carboxylic esters $\mathbf{1 7 0}$ as single diastereoisomers in excellent yield, although the reaction failed with an aromatic substituent in the $\delta$-position (Scheme 46). ${ }^{120}$ An intramolecular $\mathrm{C}-\mathrm{H}$ insertion reaction was applied in the total synthesis of $(+)$ przewalskin $\mathrm{B}$, whereby the diazo compound $\mathbf{1 7 1}$ gave the spiro-2-cyclopentenone derivative $\mathbf{1 7 2}$ in good yield when treated with rhodium(II) acetate. ${ }^{121}$
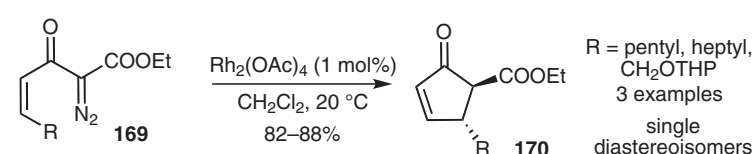

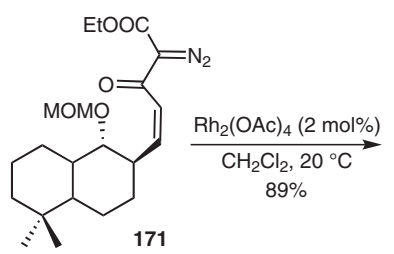<smiles>CCOC(=O)C1C(=O)C=CC12CCC1C(CCCC1(C)C)[C@@H]2OC</smiles>

Scheme 46

\section{Transformations of Existing Cyclic Systems}

As stated in the introduction, this review will not cover the extensive work done on the transformation of existing cyclopentanoid rings. However, there are some approaches to the title compound family that involve the reorganization of other, non-cyclopentane, ring systems. Two distinct categories of reactions can be identified. In one case, some masked chemical reactivity of the cyclic starting material is revealed upon treatment with a specific reagent. Often this implies a 1,4-dicarbonyl intermediate, which undergoes aldolization or crotonization, so there is some overlap with reactions discussed in the section above. In the second case, energetic factors are at play in the rearrangement of the ring system, either due to inherent ring strain or photochemical activation.

\section{Rearrangements of Furans and Pyrans}

Furans are an excellent source of 1,4-dicarbonyl compounds, and the literature on the preparation of these species and their transformations into 2-cyclopentenones up to the mid-1990s were reviewed extensively. ${ }^{122}$ Furans may be conveniently transformed into pyranones via the Achmatowicz reaction. Alternatively, sugars provide a facile entry to diverse pyran systems.

2-Furylcarbinols undergo isomerization to 4-hydroxy-2cyclopentenones upon treatment with either Brønsted or Lewis acids, a process sometimes referred to as the Piancatelli rearrangement. The accepted mechanism involves a series of cationic intermediates, and a final $4 \pi-$ electron ring closure, ${ }^{123}$ which ensures a trans configuration for the substituent at the 5-position (Scheme 47). In some cases, the acid medium (or more conveniently, basic treatment) induces further rearrangement to the more thermodynamically stable 2-substituted 4-hydroxy-2-cyclopentenones. Applications of such procedures continue to appear as part of multi-step syntheses. ${ }^{124}$

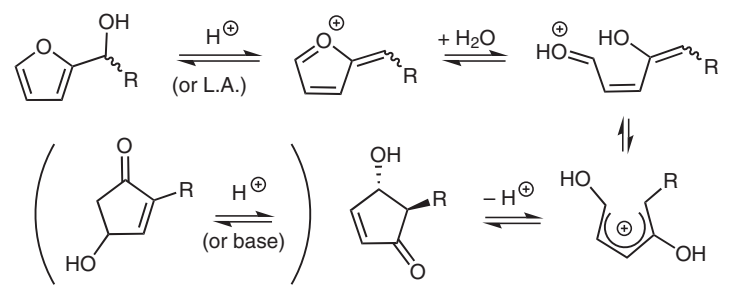

Scheme 47

In a large-scale $(>0.1 \mathrm{~mol})$ synthesis of 2-normisoprostol, a 2-furyl carbinol with an esterified alkyl chain 173 was transformed into the 4,5-disubstituted 2-cyclopentenone 174 (Scheme 48). Although the ester function was hydrolyzed during the reaction and the diastereoselectivity was not established, the yield was essentially quantitative, which was the key feature required of the transformation. ${ }^{125}$ The rearrangement of a series of representative furan substrates $\mathbf{1 7 5}$ was examined in water under microwave conditions $\left(210^{\circ} \mathrm{C}, 15\right.$ bar $)$ without added catalyst: providing the substrate had some miscibility with water, reaction times of no more than 15 minutes were required for good conversions into the corresponding 2cyclopentenones $\mathbf{1 7 6}$ with excellent anti diastereoselectivity. ${ }^{126}$

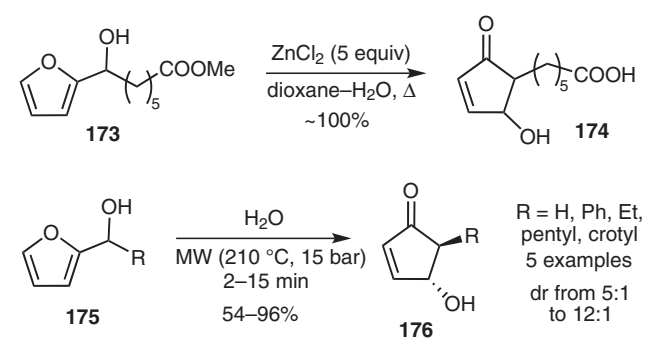

Scheme 48

Furandiols 177 were converted into fused 2-cyclopentenones 178 using a catalytic amount of Lewis acid in aqueous glyme (Scheme 49). ${ }^{127}$ The probable mechanism for this transformation was an acid-induced spiroketal formation, followed by hydrolysis to a 2-ene-1,4-dione. Intramolecular aldol condensation of this intermediate followed by intramolecular conjugate addition and finally dehydration furnished the bicyclic products. 


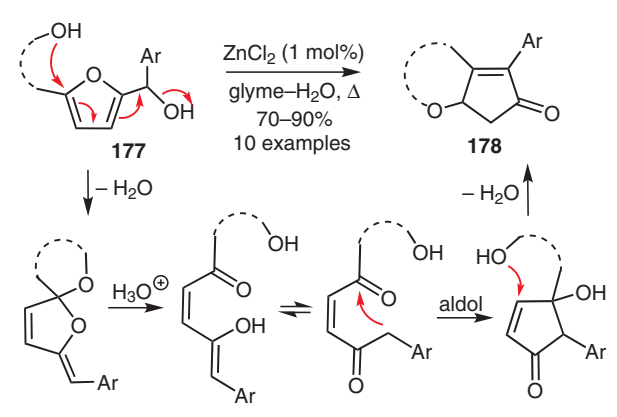

Scheme 49

An aza-Piancatelli rearrangement was established, whereby the treatment of 2-furylcarbinols $\mathbf{1 7 9}$ with an aniline in the presence of dysprosium(III) triflate as a catalyst provided a mild and direct method for the preparation of 4arylamino-2-cyclopentenones $\mathbf{1 8 0}$ (Scheme 50). Only trans adducts were obtained and yields were mostly excellent, compromised only occasionally by a competing Friedel-Crafts alkylation of electron-rich anilines. ${ }^{128} \mathrm{Re}-$ cently, aza-Piancatelli rearrangements were described using as little as $0.03 \mathrm{~mol} \%$ of phosphomolybdic acid as the catalyst. $^{129}$

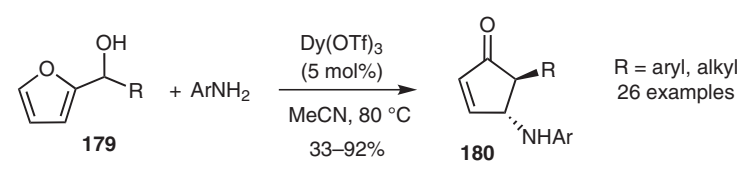

Scheme 50

In related developments in this area, 2-furaldehyde $\mathbf{1 8 1}$ was treated with two equivalents of a secondary amine in the presence of a lanthanide(III) triflate to provide very high yields of the corresponding 4,5-diamino-2-cyclopentenones 182, free of the more thermodynamically stable 2,4-isomers (Scheme 51). Here again, a concerted ringclosure step was invoked to explain the exclusively trans stereoselectivity. ${ }^{130}$ Furans bearing donor-acceptor cyclopropane substituents $\mathbf{1 8 3}$ have also been used as substrates for the aza-Piancatelli rearrangement. The reaction proceeded with either primary or secondary anilines to provide the functionalized 2-cyclopentenones 184 in good yields. Excellent diastereoselectivity was also observed, except when an electron-poor aryl group was present on the cyclopropane substrate. ${ }^{131}$

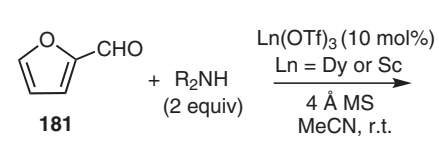
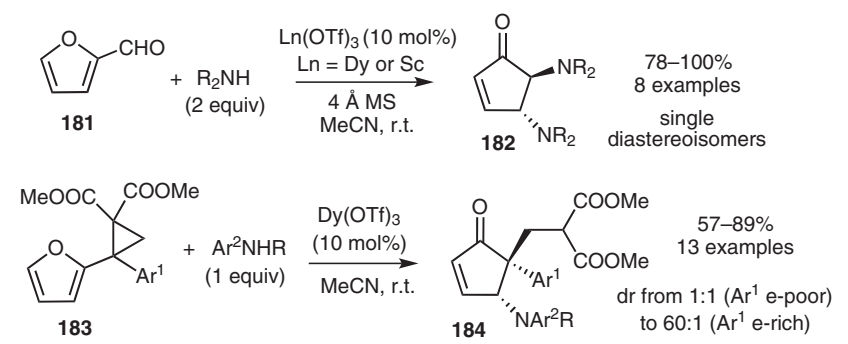

Scheme 51
An unprecedented rearrangement was reported for a polyfunctional tetrahydrofuran 185 which was transformed into cyclopentenone $\mathbf{1 8 6}$ simply upon standing for five days (Scheme 52). A mechanism involving an acidinduced series of intramolecular rearrangements proceeding via an acyclic pentadienal intermediate was proposed. ${ }^{132}$

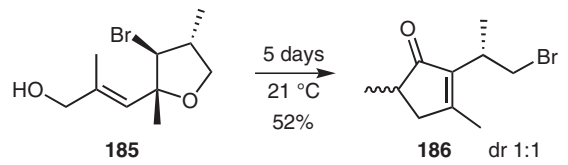

Scheme 52

The mild amine-catalyzed rearrangement of pyranones 187 provided trans-4,5-dioxygenated 2-cyclopentenones 188, a reaction which was best explained in terms of an electrocyclic ring opening followed by a conrotatory $4 \pi$ electron cyclization (Scheme 53). ${ }^{133}$ A similar ring-contraction process had been observed previously for some C2 and C4 ulopyranosides. ${ }^{134}$

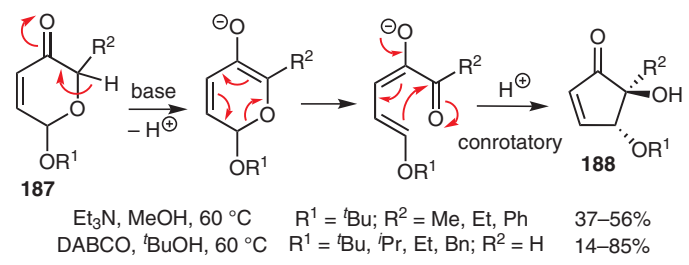

Scheme 53

During the total syntheses of guanacaterenes A and E, a cyanohydrin lactone 189, obtained as a mixture of four diastereoisomers in several steps from $(+)$-carvone, was treated with an excess of lithium hexamethyldisilazide to provide a single isomer of a 2-hydroxy-2-cyclopentenone 190 in acceptable yield (Scheme 54). The proposed mechanism involved intramolecular attack of the nitrile-stabilized anion on the lactone to form an epoxyalkoxide, which then rearranged with expulsion of cyanide to give a 1,2-diketocyclopentane which tautomerized to the most stable enone structure. ${ }^{135}$

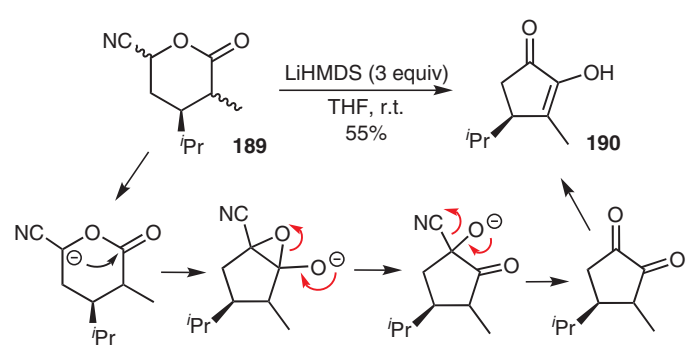

Scheme 54

A simple acid-mediated transformation of 3-methoxycarbonyl-2-methoxydihydropyrans 191 into the corresponding 3-methoxycarbonyl-2-cyclopentenones 192 was discovered (Scheme 55). The proposed mechanism, sup- 
ported by an isotopic labelling study, implied the initial elimination of methanol to give a $2 \mathrm{H}$-pyran which underwent electrocyclic $6 \pi$ ring-opening to a dienal ester. Prins cyclization followed by elimination of water and isomerization gave the products in moderate yields. ${ }^{136}$

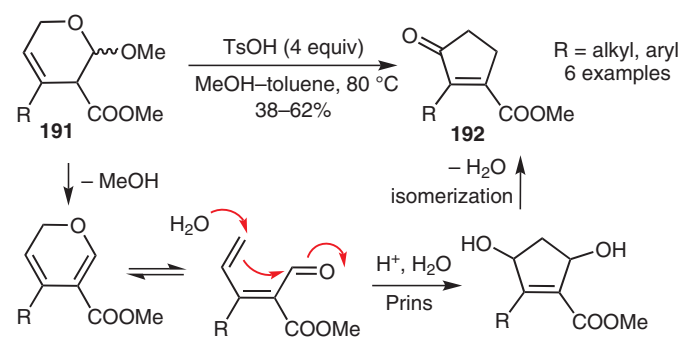

Scheme 55

A novel aza-variant of the pyran-ring-contraction approach has been established for the reaction of 4,6-dialkylglycals 193 with aromatic amines in the presence of indium bromide, to give trans-2,5-dialkyl-4-arylamino-2cyclopentenones 194 in fair to good yields (Scheme 56). A mechanism was proposed, whereby a Ferrier-type reaction produced a cyclic aminal intermediate which underwent indium-mediated ring opening, dehydration, then conrotatory $4 \pi$-electron ring closure. ${ }^{137}$

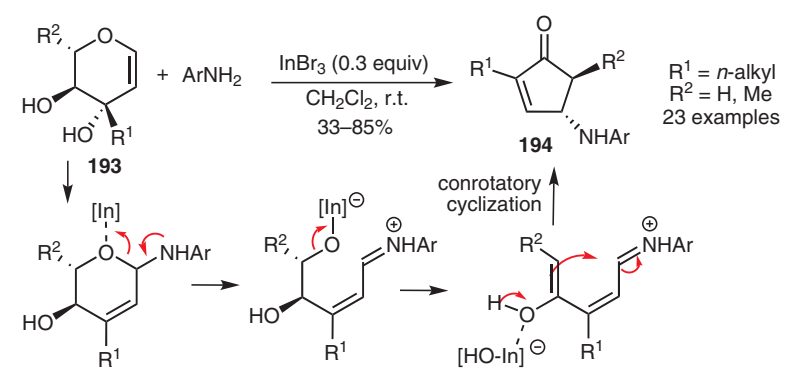

Scheme 56

\section{Reactions of Sugar Lactones}

Over the years, ribosides and ribonolactone derivatives have been popular precursors for the preparation of 4,5dioxygenated 2-cyclopentenones in single enantiomeric form. As part of a synthesis of carbon-substituted nucleosides, a Wittig reagent was added to the silyl enol ether 195 to generate an intermediate diketophosphorus ylide 196, which cyclized to provide the highly substituted 2cyclopentenone 197 in reasonable yield (Scheme 57). ${ }^{138}$ The enol ether 199 was prepared from a D-ribose derivative 198 by treatment with the Tebbe reagent; its treatment with dimethylaluminum chloride gave a good yield of the 2-cyclopentenone 200, via a Lewis acid induced isopropyl group cleavage followed by an intramolecular aldol reaction. It had been hoped that the Tebbe reagent might induce the rearrangement and thus provide a cascade sequence towards some pentenomycin targets; in the event, dimethylaluminum chloride was the only Lewis acid able to induce the rearrangement, and even this failed with the corresponding compound lacking the hydroxymethyl group at C5. ${ }^{139}$ An interesting tandem process was developed in which allylic substrates 201 were isomerized using photochemically activated iron(0) carbonyl to generate silyl ketal enol ethers 202, which were treated with fluoride to induce ring opening followed by an intramolecular aldol reaction, to provide the 2-cyclopentenones 203. ${ }^{140}$

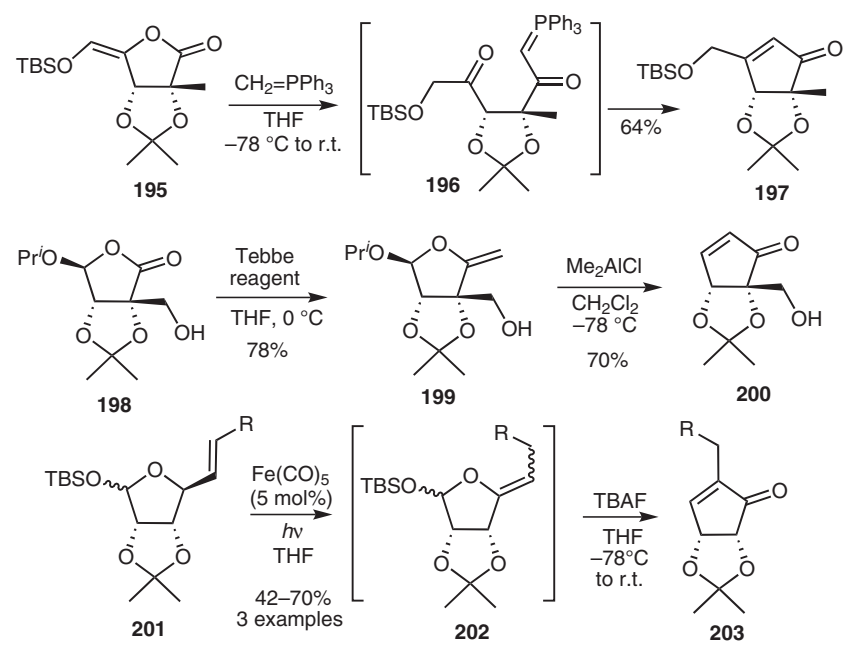

Scheme 57

\section{Ring Contractions}

The photorearrangement of cyclohexa-2,5-dienones to bicyclo[3.1.0] hex-3-en-2-ones is a well-studied transformation, and was used to carry out formal syntheses of $( \pm)$ methyleneomycins A and B (Scheme 58). The masked $p$ benzoquinones 204 were irradiated to furnish 2-cyclopentenones $\mathbf{2 0 5}$ or $\mathbf{2 0 6}$ with a carboxylic ester in either the 4or 5-position, respectively. After the usual di- $\pi$-methane rearrangement from the excited state, the regioselectivity of the three-membered-ring bond fission of the bicyclic intermediate depends on the presence of a bridgehead substituent. $^{141}$

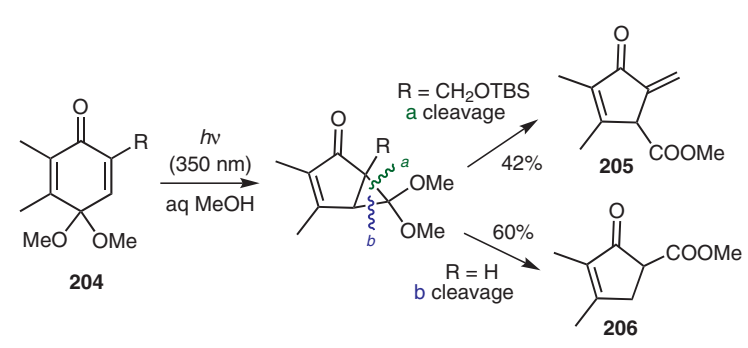

Scheme 58

Rearrangements of cyclohexa-2,4-dienones may also lead to 2-cyclopentenones. When solutions of selected masked $o$-benzoquinones $\mathbf{2 0 7}$ in chloroform were treated with singlet oxygen followed by thiourea (a reducing agent), the anticipated endoperoxides were unexpectedly accompanied by 4-hydroxy-2-cyclopentenones 208; these latter products were the only ones obtained when the reactions 
were run in methanol (Scheme 59). This ring contraction was proposed to occur via initial formation of the perepoxide intermediate, which underwent an unusual [1,2]acyl shift in a highly stereoselective manner. ${ }^{142}$

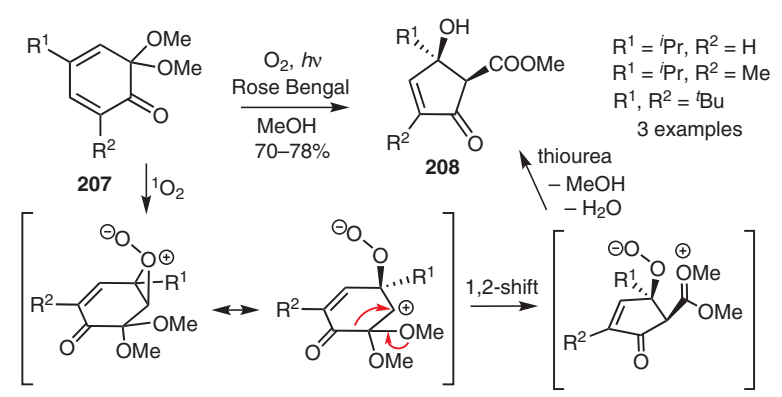

Scheme 59

\section{Ring Expansions}

Cyclopropanes and cyclobutanes have considerable ring strain, so rearrangements involving expansion to a fivemembered ring are energetically favored. Some reviews on small-ring chemistry have covered formation of 2-cyclopentenones (amongst other compounds) via these routes. ${ }^{143}$

A popular way of conducting a cyclobutanone ring expansion is to prepare the corresponding spiroepoxide, then rearrange it using a Lewis acid. A suitably placed leaving group is necessary for the extra unsaturation present in 2cyclopentenones (Scheme 60). After some optimization studies with a small series of 2-ethoxycyclobutanones, the chiral derivative $\mathbf{2 0 9}$ was treated with dimethylsulfoxonium methylide to generate a single spiroepoxide which was efficiently ring-expanded in a one-pot procedure using scandium triflate to give the cyclopentanone 210. Treatment with base was necessary to eliminate ethanol, and the resulting 2-cyclopentenone $\mathbf{2 1 1}$ was then used in the syntheses of $(+)$-carbovir and $(+)$-aristeromycin. ${ }^{144} \mathrm{~A}$ selection of protected 2-aminocyclobutanones 212 were transformed into the corresponding spiroepoxides 213 using dimethylsulfonium methylide. Each of these was smoothly converted into the corresponding 2-cyclopentenone 214 upon treatment with lithium iodide, via ring expansion and spontaneous $\beta$-elimination of the amide fragment. Yields were generally high and the transformation proceeded with no loss of the stereochemical information derived from the cyclobutanone substrate. ${ }^{145}$

An interesting regioselectivity issue was uncovered during studies on the ring expansion of 2-hydroxycyclobutanone aldol adducts $\mathbf{2 1 5}$, using acidic ion-exchange resin (Scheme 61). With no other ring substituents, the skeletal rearrangement gave the 3-hydroxy-2-cyclopentenone 216, which is a tautomer of the cyclic 1,3-diketone. On the other hand, with the corresponding 3,3-dimethylated cyclobutanones, the acid-mediated rearrangement gave the 2-hydroxy-2-cyclopentenone regioisomers $217 .{ }^{146}$ These reaction profiles were interpreted in terms of the relative migratory aptitudes of the acyl and alkyl groups involved.
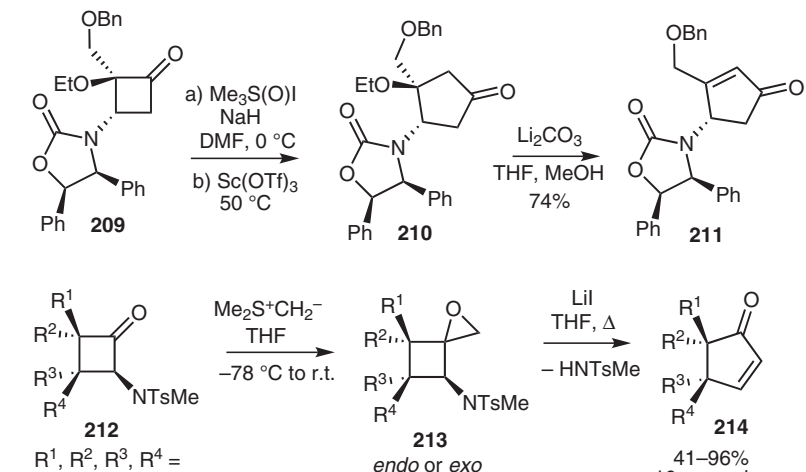
$\mathrm{Me}_{2} \mathrm{~S}^{+} \mathrm{CH}_{2}^{-}$ $\underset{-78^{\circ} \mathrm{C} \text { to r.t. }}{\stackrel{\mathrm{THF}}{\longrightarrow}}$
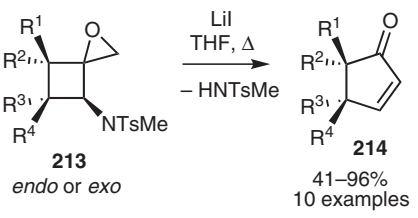

$\mathrm{H}$, alkyl (often cyclic)

\section{Scheme 60}
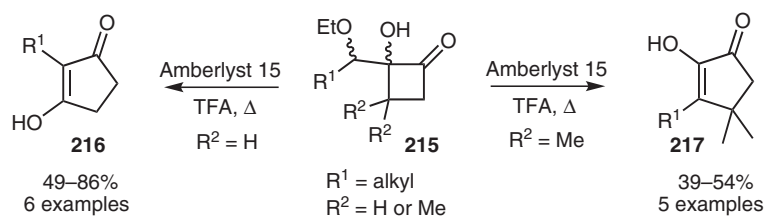

Scheme 61

2,2-Dichlorocyclobutanes react with diazomethane via a regioselective ring-expansion followed by dehydrochlorination to give the 2-chloro-2-cyclopentenone framework. This reactivity was exploited to transform the dichloroketene adduct of 7-methylcycloheptatriene 218 into a single hydroazulenone 219 in $76 \%$ yield (Scheme 62). ${ }^{147}$ This compound was a key intermediate in the total synthesis of $( \pm)$-6-deoxygeigerin and, later, of $( \pm)$-geigerin. ${ }^{148}$
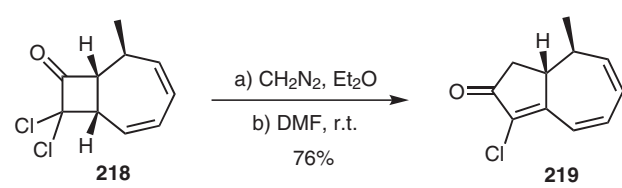

\section{Scheme 62}

The unique chemistry of squaric acid and its derivatives has been exploited for selective ring expansions to give 2cyclopentenones (Scheme 63). Double addition of vinyl magnesium bromide to the derivatives $\mathbf{2 2 0}$ proceeded in a 1,2-/1,4-fashion to give linear octatetraene species $\mathbf{2 2 1}$, which upon selective protonation during work-up underwent cyclization to give the polyfunctional derivatives 222 in good yield in a one-pot operation. ${ }^{149}$

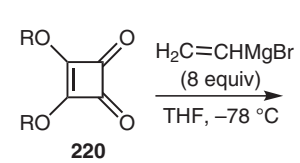

$\mathrm{R}=\mathrm{Me},{ }^{\mathrm{i}} \mathrm{Pr}, \mathrm{Bu}$

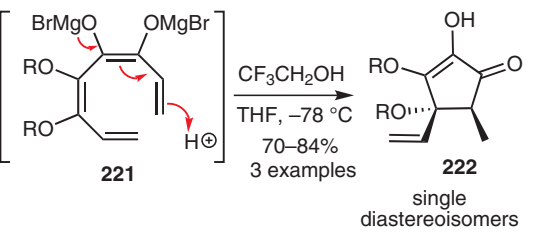

Scheme 63 
A 'squarate ester cascade' protocol has been used to construct angular or linear triquinane sequiterpene skeletons in an elegant fashion (Scheme 64). Sequential addition of two alkenyllithium species to diisopropyl squarate $\mathbf{2 2 3}$ provided, irrespective of the geometry of the dienolate adduct, an intermediate which evolved first via eightmembered-ring closure to dienolate $\mathbf{2 2 4}$ then further via intramolecular aldol closure to give the linear tricyclic structure 225 in 24\% yield (after acidic hydrolysis of the enol). ${ }^{150}$ On the other hand, the successive addition of an alkenyllithium then an alkynyllithium to $\mathbf{2 2 3}$ proceeded via a helical dienolate which ring-closed via a strained intermediate structure 226; the latter then evolved via intramolecular enolate attack to close the fused five-membered ring of 227, in $68 \%$ yield. These ring-assembly protocols were exploited for the total synthesis of sesquiterpenes.
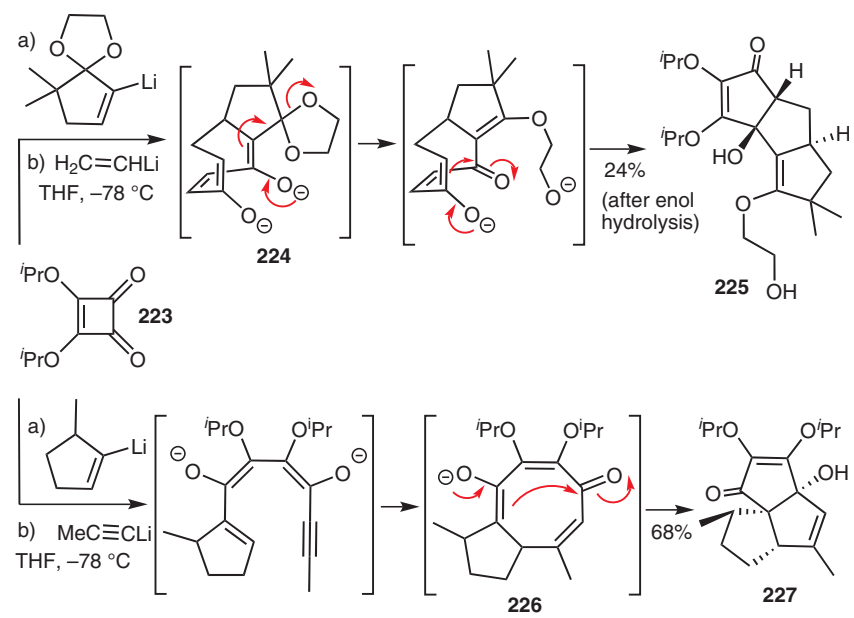

Scheme 64

1-Vinyl-1-silyloxy-2,2-dichlorocyclopropanes 228, easily prepared from silyloxydienes, were treated with a sil$\operatorname{ver}(\mathrm{I})$ reagent to induce a sequential $2 \pi$ ring opening and $4 \pi$-electron ring closure, the latter step being analogous to the Nazarov cyclization (Scheme 65). This process provided chloro-substituted 2-cyclopentenones 229 and 230 in variable proportions and reasonable yields, albeit with limited control of regio- and diastereoselectivities. ${ }^{151}$
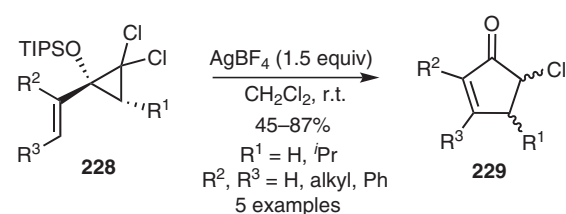

229 5 examples

Scheme 65

The ring expansion of alkynylcyclopropanols 231 to provide 3 -substituted 2-cyclopentenones 232 was achieved using a ruthenium catalyst in the presence of indium triflate and a Brønsted acid (Scheme 66). The reaction was successful with alkyl-substituted alkynes, for which an insertion mechanism via a ruthenium metallacycle was sug- gested. With more electron-deficient alkynes, notably acyl derivatives, the reaction evolved differently to give 2-vinylcyclobutanones. ${ }^{152}$

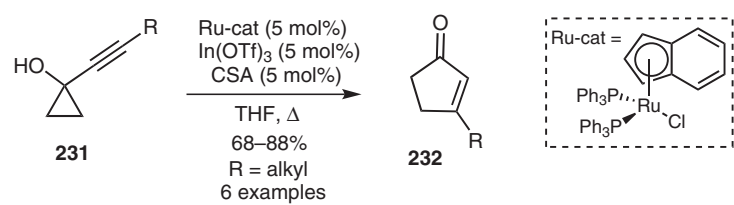

Scheme 66

A ring expansion of highly substituted epoxides bearing acetate and propargylic ester functions $\mathbf{2 3 3}$ was achieved using a platinum catalyst (Scheme 67). Even though syn/anti mixtures of the substrates were employed, the product 2-cyclopentenones 234 were obtained as single diastereoisomers. The proposed mechanism involved evolution of a metal-alkyne complex with acetate migration to form a platinum carbene, which reacted with the epoxide to form a pyran $\mathbf{2 3 5}$. An unprecedented tandem oxa- $6 \pi$ ring opening followed by platinum-mediated conrotatory $4 \pi$-electron ring closure accompanied by acetate transposition was proposed to explain the transformation of the pyran to give the final product $234 .{ }^{153}$ This reaction is clearly related to the Nazarov and Rautenstrauch cyclization reactions (vide supra).

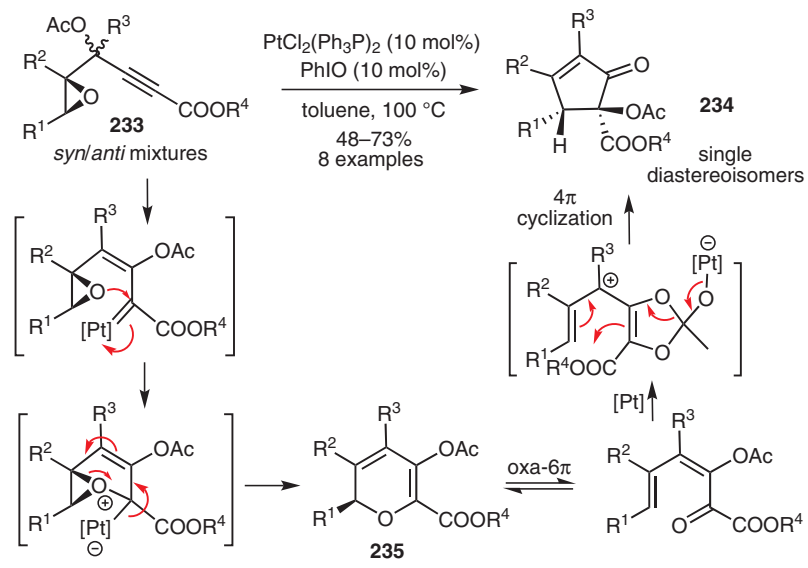

Scheme 67

The treatment of the vicinal diol 236 with strong base provided the 2-cyclopentenone 237 , reportedly in good yield (Scheme 68). ${ }^{154}$ The transformation is noteworthy as the first (and so far only) apparent example of an anionic oxy retro-ene reaction, giving in this case a diketone enolate which evolved by intramolecular aldol condensation.

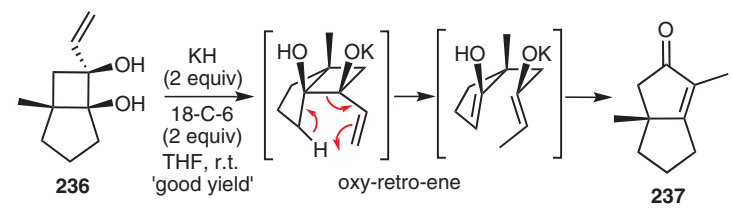

Scheme 68 
Finally, 1,1-disubstituted spiropentanes $\mathbf{2 3 8}$ were converted into 2-cyclopentenones $\mathbf{2 3 9}$ in the presence of carbon monoxide and a rhodium catalyst (Scheme 69). The proposed mechanism involved evolution through a series of rhodacycles of increasing size, so that one of the original spiropentane carbons ended up as a methyl substituent in an exocyclic locus in the final structure. ${ }^{155}$ Two examples of cyclic-fused 1,2-disubstituted spiropentanes also evolved in a similar fashion with retention of the ring junction stereochemistry, albeit in slightly lower yield.

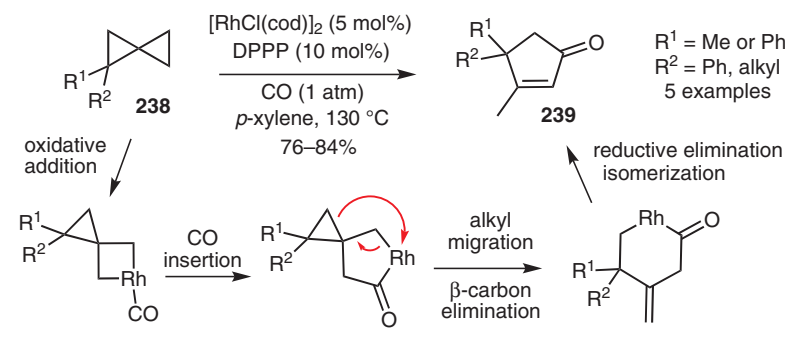

Scheme 69

\section{5}

\section{Miscellaneous Methods}

A few reports of 2-cyclopentenone preparation cannot be conveniently classed in any of the above sections and are therefore collected in this final section.

The retro-Diels-Alder strategy for the preparation of substituted 2-cyclopentenones from the tetrahydro-4,7-methano-1-indanone skeleton was established some time ago, but the cracking conditions required to liberate the target structures can be somewhat restrictive. Nevertheless, an attractive feature of this approach is the relative ease of access to the starting materials. The approach therefore continues to enjoy some synthetic applications (Scheme 70). The preparation of the tri-substituted 2-cyclopentenone 241, a key building block for a proposed total synthesis of the diterpene antibiotic guanacasterpene A, was achieved via a flash vacuum pyrolysis (FVP) retro-DielsAlder reaction of $\mathbf{2 4 0}$ in excellent yield. ${ }^{156}$ In the synthesis of selected phytoprostanes for evaluation as PPAR- $\gamma$ ligands, a retro-Diels-Alder reaction was performed on tricyclic adduct 242 under mild conditions employing a Lewis acid and an excess of maleic anhydride to trap the cyclopentadiene by-product. The $E$-isomer of the 2-cyclopentenone product 243 predominated, regardless of the configuration $(Z$ or $E)$ of the substrate. ${ }^{157}$ Similarly, the use of zeolites serving as heterogeneous Brønsted acids facilitated the transformation of a series of tricyclic substrates 244 into 4-alkyl-2-cyclopentenones 245 under mild conditions. ${ }^{158}$

The tandem [2+2]-cycloaddition-Dieckmann condensation of $\gamma$-keto esters 246 with lithium ynolates gave, in the first instance, bicyclic $\beta$-lactones 247 , which readily eliminated carbon dioxide upon heating to provide 2,3-disubstituted 2-cyclopentenones $\mathbf{2 4 8}$ in a one-pot process (Scheme 71). ${ }^{159}$ An application of this protocol was made

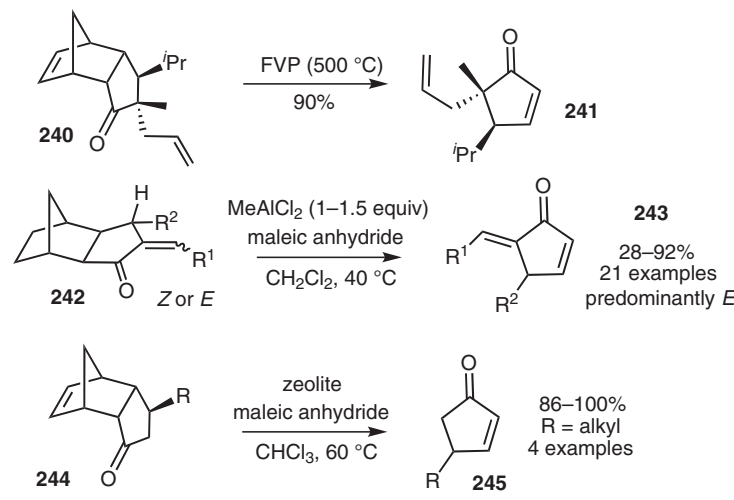

Scheme 70

in the final step of a total synthesis of the triquinane natural product cucumin E (250) by transformation of the bicyclic precursor 249. ${ }^{160}$

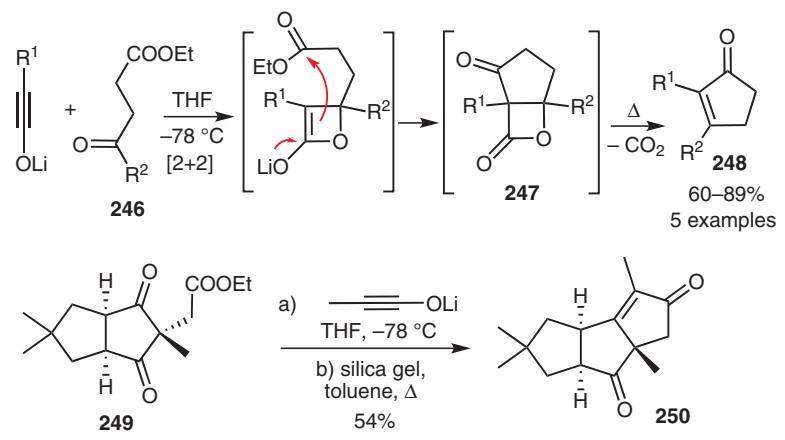

Scheme 71

In a study of synthetic routes to prostanoids, an unusual tandem reaction process was discovered when selected aldehydes 251 were transformed into the pyrrolidine enamines and heated with 3-iodo-2-methoxymethoxy-1propene. 4,4-Disubstituted 2-cyclopentenones 252 were formed directly, albeit in moderate yields, in what appeared to be an original domino aza-Claisen-Mannich cyclization, terminating in $\beta$-elimination of the secondary amine (Scheme 72). In several cases, the aldehyde substrate was a chiral sugar derivative but the spiro adducts were obtained without significant diastereomeric excesses. ${ }^{161}$

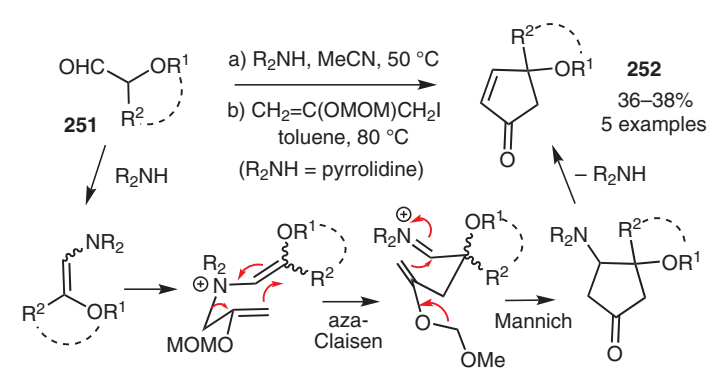

Scheme 72 
4-Alkylidene-2-cyclopentenones 254 were prepared from unsaturated 1,4-diketones $\mathbf{2 5 3}$ and selected nitroalkanes in a mild tandem ring-closure-Michael addition-elimination process, with good yields and excellent $E$-stereoselectivity (Scheme 73). ${ }^{162}$ This is a surprisingly simple yet attractive route to the target compounds which can otherwise be difficult to prepare.

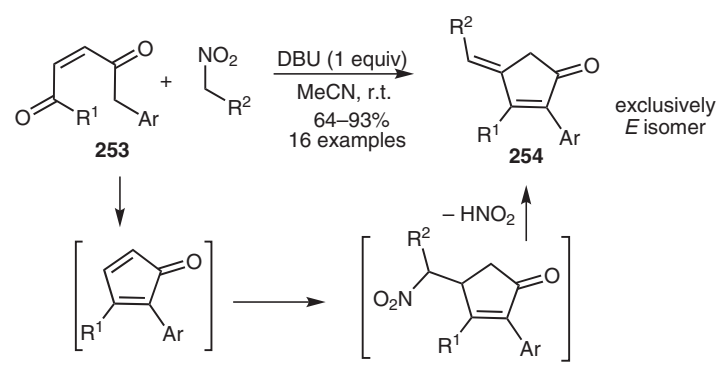

Scheme 73

An intriguing cascade reaction combining benzil (255) and two equivalents of an alkynyllithium has been described, in which it was proposed that the initial 1,5hexadiyn-3,4-olate adduct evolved via an anionic oxyCope rearrangement then an intramolecular aldol condensation and a [1,2]-aryl shift, to give 2-phenyl-5-benzoyl2-cyclopentenones 256 (Scheme 74). The reaction was substrate-dependent, for competing reaction pathways were evidenced, but several examples employing an aryl or alkyl acetylide proceeded in high yield. The 5-acyl-2cyclopentenone products exhibited the expected ketoenol tautomeric equilibria. ${ }^{163}$

Finally, an unprecedented cyclotrimerization of $\alpha$-monosubstituted aldehydes induced by dibromotriphenylphosphine has been described (Scheme 75). ${ }^{164}$ The proposed mechanism consisted of a series of Lewis acid mediated aldol condensations going via a propenal to give a pentadienal, which isomerized before cyclizing via a Nazarovtype process to give the 2,3,5-trisubstituted 2-cyclopent- enone products 257 . On this postulate, mixed condensations of aldehydes and enals were carried out using the same conditions to give the expected products $\mathbf{2 5 8}$.
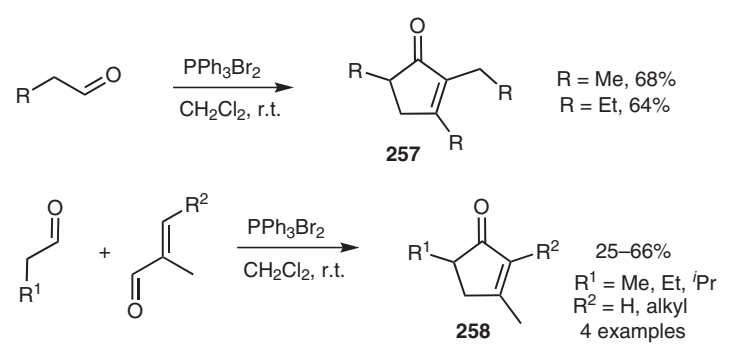

Scheme 75

\section{Conclusions}

As this review shows, an ever-expanding array of synthetic methodologies is available for the synthesis of 2-cyclopentenones. Established reactions continue to be further developed while new approaches continue to emerge. Some developments emphasize the use of catalytic processes or benign reaction conditions, while others prioritize selectivity issues or other practicalities. A significant generality to emerge from this overview is that there is no one reaction which dominates the scene: the 'best' choice of synthesis for any given target will depend on a balance of many factors. Given the enduring importance of the title compounds and the efforts currently geared towards their synthesis, it seems likely that methodologies will continue to develop at a considerable pace.

\section{Acknowledgment}

One of us (H.E.) was the beneficiary of a PhD grant (French MESR Allocation) earmarked by Université Paris-Sud for bilateral international collaboration. The authors are grateful to the Ile-de-France region for travel funding (SETCI exchange programme).

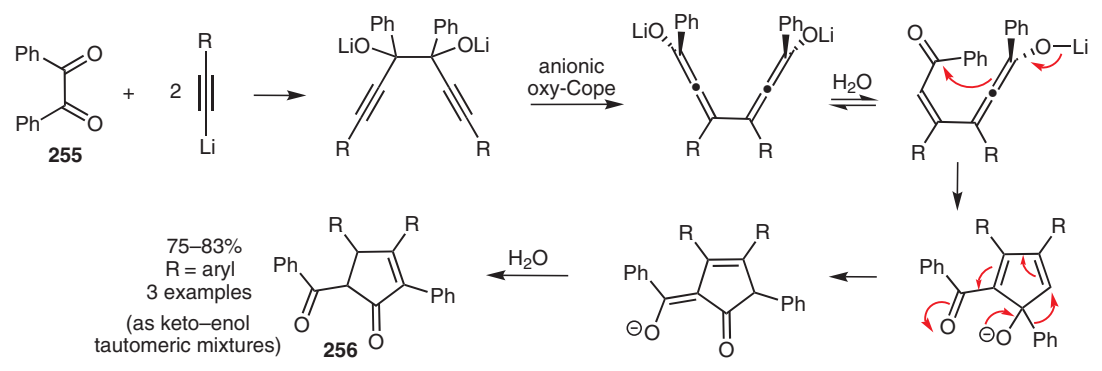

Scheme 74 


\section{References}

(1) (a) Gibson, S. E.; Lewis, S. E.; Mainolfi, N. J. Organomet. Chem. 2004, 689, 3873. (b) Roche, S. P.; Aitken, D. J. Eur. J. Org. Chem. 2010, 5339. (c) Mehta, G.; Srikrishna, A. Chem. Rev. 1997, 97, 671. (d) Hudlicky, T.; Price, J. D. Chem. Rev. 1989, 89, 1467. (e) Trost, B. M. Chem. Soc. Rev. 1982, 11, 141. (f) Marsden, S. P. Science of Synthesis; Vol. 26; Cossy, J., Ed.; Thieme: Stuttgart, 2004, 1045.

(2) Ellison, R. A. Synthesis 1973, 397.

(3) Coote, S. C.; O'Brien, P.; Whitwood, A. C. Org. Biomol. Chem. 2008, 6, 4299.

(4) Basavaiah, D.; Reddy, B. S.; Badsara, S. S. Chem. Rev. 2010, 110, 5447.

(5) Declerck, V.; Martinez, J.; Lamaty, F. Chem. Rev. 2009, 109, 1 .

(6) Koech, P. K.; Krische, M. J. J. Am. Chem. Soc. 2004, 126, 5350.

(7) Krafft, M. E.; Cran, J. W. Synlett 2005, 1263.

(8) (a) Smith, A. B. III; Branca, S. J.; Guaciaro, M. A.; Wovkulich, P. M.; Korn, A. Org. Synth. 1983, 61, 65. (b) Kim, K. M.; Chung, K. H.; Kim, J. N.; Ryu, E. K. Synthesis 1993, 283. (c) Adam, W.; Saha-Moeller, C. R.; Zhao, C.-G. Org. React. 2002, 61, 219.

(9) (a) Negishi, E.-i. Tetrahedron 2000, 56, 10197. (b) Miller, M. W.; Johnson, C. R. J. Org. Chem. 1997, 62, 1582. (c) Dyker, G.; Markwitz, H.; Henkel, G. Eur. J. Org. Chem. 2001, 2415. (d) Molander, G. A.; Ham, J.; Seapy, D. G. Tetrahedron 2007, 63, 768.

(10) Liu, K.-M.; Chau, C.-M.; Sha, C.-K. Chem. Commun. 2008, 91.

(11) Cho, D. J.; Wu, C. J.; S, S.; Han, W.-S.; Kang, S. O.; Lee, B. Y. Organometallics 2006, 25, 2133.

(12) (a) Morisaki, Y.; Imoto, H.; Hirano, K.; Hayashi, T.; Chujo, Y.J. Org. Chem. 2011, 76, 1795. (b) Thaler, T.; Guo, L.-N.; Steib, A. K.; Raducan, M.; Karaghiosoff, K.; Mayer, P.; Knochel, P. Org. Lett. 2011, 13, 3182. (c) Wei, C.-H.; Mannathan, S.; Cheng, C.-H. J. Am. Chem. Soc. 2011, 133, 6942. (d) Piovesana, S.; Scarpino Schietroma, D. M.; Tulli, L. G.; Monaco, M. R.; Bella, M. Chem. Commun. 2010, 46, 5160. (e) Sibi, M. P.; Manyem, S. Tetrahedron 2000, 56, 8033.

(13) (a) Perdicchia, D.; Jørgensen, K. A. J. Org. Chem. 2007, 72, 3565. (b) Yang, L.; Xu, L.-W.; Zhou, W.; Li, L.; Xia, C.-G. Tetrahedron Lett. 2006, 47, 7723. (c) Rana, N. K.; Selvakumar, S.; Singh, V. K. J. Org. Chem. 2010, 75, 2029.

(14) (a) Krishna, T. R.; Jayaraman, N. Tetrahedron 2004, 60, 10325. (b) Li, J.-H.; Wang, D.-P.; Xie, Y.-X. Tetrahedron Lett. 2005, 46, 4941.

(15) Guo, H.-C.; Ma, J.-A. Angew. Chem. Int. Ed. 2006, 45, 354

(16) Gerdil, R.; Liu, H.; Bernardinelli, G. Helv. Chim. Acta 1999, $82,418$.

(17) (a) Kelly, J. M.; Leeper, F. J. Tetrahedron Lett. 2012, 53, 819. (b) Tolstikov, G. A.; Miftakhov, M. S.; Danilova, N. A.; Vel'der, Ya. L.; Spirikhin, L. V. Synthesis 1989, 625.

(18) (a) Demir, A. S.; Enders, D. Tetrahedron Lett. 1989, 30, 1705. (b) Mukaiyama, T.; Ohsumi, T. Chem. Lett. 1983, 875. (c) Lakhvich, F. A.; Lis, L. G.; Pap, A. A.; Rubinov, D. B.; Zheldakova, T. A.; Akhrem, A. A. Zh. Org. Khim. 1988, 24,518 .

(19) (a) Schulé, A.; Liang, H.; Vors, J.-P.; Ciufolini, M. A. J. Org. Chem. 2009, 74, 1588. (b) Altenbach, R. J.; Brune, M. E.; Buckner, S. A.; Coghlan, M. J.; Daza, A. V.; Fabiyi, A.; Gopalakrishnan, M.; Henry, R. F.; Khilevich, A.; Kort, M. E.; Milicic, I.; Scott, V. E.; Smith, J. C.; Whiteaker, K. L.; Carroll, W. A. J. Med. Chem. 2006, 49, 6869.

(20) (a) Kobayashi, Y.; Murugesh, M. G.; Nakano, M.; Takahisa, E.; Usmani, S. B.; Ainai, T. J. Org. Chem. 2002, 67, 7110. (b) Weaving, R.; Roulland, E.; Monneret, C.; Florent, J.-C. Tetrahedron Lett. 2003, 44, 2579. (c) Shi, M.; Zhang, W. Tetrahedron 2005, 61, 11887.

(21) (a) Mizuno, M.; Inoue, H.; Naito, T.; Zhou, L.; Nishiyama, H. Chem. Eur. J. 2009, 15, 8985. (b) Das, J.; Le Cavelier, F.; Rouden, J.; Blanchet, J. Eur. J. Org. Chem. 2011, 6628.

(22) (a) Pete, J.-P. In CRC Handbook of Organic Photochemistry and Photobiology, 2nd ed.; Horspool, W.; Lenci, F., Eds.; CRC Press: Boca Raton, 2003, Chap. 71, 1-14. (b) Le Liepvre, M.; Ollivier, J.; Aitken, D. J. Eur. J. Org. Chem. 2009, 5953. (c) Margaretha, P. In Synthetic Organic Photochemistry; Griesbeck, A. G.; Mattay, J., Eds.; Marcel Dekker: New York, 2005, Chap. 8, 211-237.

(23) (a) Xu, H.; Wolf, C. Angew. Chem. Int. Ed. 2011, 50, 12249. (b) Canales, E.; Corey, E. J. Org. Lett. 2008, 10, 3271. (c) Northrup, A. B.; MacMillan, D. W. C. J. Am. Chem. Soc. 2002, 124, 2458

(24) (a) Henry, S. S.; Brady, M. D.; Laird, D. L. T.; Ruble, J. C.; Varie, D. L.; Monn, J. A. Org. Lett. 2012, 14, 2662.

(b) Garcia Ruano, J. L.; Alonso, M.; Cruz, D.; Fraile, A.; Martin, M. R.; Peromingo, M. T.; Tito, A.; Yuste, F. Tetrahedron 2008, 64, 10546.

(25) Menjo, Y.; Hamajima, A.; Sasaki, N.; Hamada, Y. Org. Lett. 2011, 13, 5744.

(26) Lee, A.; Reisinger, C. M.; List, B. Adv. Synth. Catal. 2012, $354,1701$.

(27) (a) Schore, N. E. Org. React. 1991, 40, 1. (b) Geis, O.; Schmalz, H.-G. Synthesis 1998, 911. (c) Brummond, K. M.; Kent, J. L. Tetrahedron 2000, 56, 3263. (d) Laschat, S.; Becheanu, A.; Bell, T.; Baro, A. Synlett 2005, 2547. (e) Lee, H.-W.; Kwong, F.-Y. Eur. J. Org. Chem. 2010, 789. (f) Blanco-Urgoiti, J.; Añorbe, L.; Pérez-Serrano, L.; Domínguez, G.; Pérez-Castells, J. Chem. Soc. Rev. 2004, 33, 32.

(28) Gibson, S. E.; Mainolfi, N. Angew. Chem. Int. Ed. 2005, 44, 3022.

(29) Shibata, T. Adv. Synth. Catal. 2006, 348, 2328.

(30) The Pauson-Khand Reaction: Scope Variations and Applications; Torres, R. R., Ed.; Wiley: Chichester, 2012.

(31) For leading references, see: Wu, N.; Deng, L.; Liu, L.; Liu, Q.; Li, C.; Yang, Z. Chem. Asian J. 2013, 8, 65 .

(32) Morimoto, T.; Kakiuchi, K. Angew. Chem. Int. Ed. 2004, 43, 5580 .

(33) Hong, B. C.; Dange, N. S.; Yen, P.-J.; Lee, G.-H.; Liao, J.H. Org. Lett. 2012, 14, 5346.

(34) Su, S.; Rodriguez, E. A.; Baran, P. S. J. Am. Chem. Soc. 2011, 133, 13922.

(35) Tap, A.; Jouanneau, M.; Galvani, G.; Sorin, G.; Lannou, M.I.; Ferezou, J.-P.; Ardisson, J. Org. Biomol. Chem. 2012, 10, 8140 .

(36) Turlington, M.; Du, Y.; Oqtrum, S. G.; Santosh, V.; Wren, K.; Lin, T.; Sabat, M.; Pu, L. J. Am. Chem. Soc. 2011, 133, 11780.

(37) Morisaki, Y.; Kondo, T.; Mitsudo, T.-A. Org. Lett. 2000, 2 , 949.

(38) Huang, Q.; Hua, R. Chem. Eur. J. 2009, 13, 3817.

(39) Ohashi, M.; Taniguchi, T.; Ogoshi, S. J. Am. Chem. Soc. 2011, 133, 14900 .

(40) Barluenga, J.; Barrio, P.; Riesgo, L.; López, L. A.; Tomás, M. J. Am. Chem. Soc. 2007, 129, 14422.

(41) Barluenga, J.; Álvarez-Fernández, A.; Suárez-Sobrino, Á. L.; Tomás, M. Angew. Chem. Int. Ed. 2012, 51, 183.

(42) Qi, X.; Ready, J. M. Angew. Chem. Int. Ed. 2008, 47, 7068.

(43) Miller, J. A.; Pugh, A. W.; Ullah, G. M.; Welsh, G. M. Tetrahedron Lett. 2001, 42, 955.

(44) Tius, M. A. Acc. Chem. Res. 2003, 36, 284. 
(45) Hu, H.; Smith, D.; Cramer, R. E.; Tius, M. A. J. Am. Chem. Soc. 1999, 121, 9895.

(46) Schultz-Fademrecht, C.; Tius, M. A.; Grimme, S.; Wibbeling, B.; Hoppe, D. Angew. Chem. Int. Ed. 2002, 41, 1532.

(47) Jacobsen, C. B.; Jensen, K. L.; Udmark, J.; Jørgensen, K. A. Org. Lett. 2011, 13, 4790.

(48) Feist, H.; Langer, P. Synthesis 2008, 3877.

(49) Langer, P.; Kracke, B. Synlett 2001, 1790.

(50) Shinohara, Y.; Kurata, T.; Kitano, H.; Matsumoto, K.; Takahashi, I.; Hosoi, S.; Ota, T.; Hatanaka, M. Synlett 2002, 1245.

(51) (a) Cai, X.-h.; Xie, B. Res. J. Chem. Sci. 2011, 1, 120. (b) Marjani, K.; Mohsen, M.; Arazi, A.; Ashouri, A.; Bourghani, S.; Rajabi, M. Monatsh. Chem. 2009, 140, 1331. (c) Marjani, K.; Mousavi, M.; Ashouri, A.; Arazi, O.; Bourghani, S.; Asgari, M. J. Chem. Res. 2008, 398. (d) Majani, K.; Asgari, M.; Ashouri, A.; Mahdavinia, G. H.; Ahangar, H. A. Chin. Chem. Lett. 2009, 20, 401.

(52) Holtz, E.; Köhler, V.; Appel, B.; Langer, P. Eur. J. Org. Chem. 2005, 532.

(53) Jose, A.; Lakshmi, K. C. S.; Suresh, E.; Nair, V. Org. Lett. 2013, 15, 1858.

(54) Gagnier, S. V.; Larock, R. C. J. Am. Chem. Soc. 2003, 125, 4804.

(55) Li, H.; Liu, L.; Zhao, F.; Wang, C.; Wang, C.; Song, Q.; Zhang, W.-X.; Xi, Z. J. Org. Chem. 2012, 77, 4793.

(56) Laroche, C.; Bertus, P.; Szymoniak, J. Chem. Commun. 2005, 3030.

(57) Rigby, J. H.; Wang, Z. Org. Lett. 2003, 5, 263.

(58) Davie, C. P.; Danheiser, R. L. Angew. Chem. Int. Ed. 2005, 44, 5867.

(59) Moser, W. H.; Feltes, L. A.; Sun, L.; Giese, M.; Farrell, R. W. J. Org. Chem. 2006, 71, 6542.

(60) Giese, M.; Moser, W. H. Org. Lett. 2008, 10, 4215.

(61) Li, Z.; Moser, W. H.; Deng, R.; Sun, L. J. Org. Chem. 2007, 72,10254

(62) Kurahashi, T.; Wu, Y.-T.; Meindl, K.; Rühl, S.; de Meijere, A. Synlett 2005, 805 .

(63) Kurteva, V. B.; Afonso, C. A. M. Chem. Rev. 2009, 109, 6809.

(64) Nazarov, I. N.; Zaretskaya, I. I. Izv. Akad. Nauk. SSSR, Ser. Khim. 1944, 65.

(65) (a) Rueping, M.; Ieawsuwan, W.; Antonchick, A. P.; Nachtsheim, B. J. Angew. Chem. Int. Ed. 2007, 46, 2097. (b) Basak, A. K.; Shimada, N.; Bow, W. F.; Vicic, D. A.; Tius, M. A. J. Am. Chem. Soc. 2010, 132, 8266. (c) Bow, W. E.; Basak, A. K.; Jolit, A.; Vicic, D. A.; Tius, M. A. Org. Lett. 2010, 12, 440. (d) Rueping, M.; Ieawsuwan, W. Chem. Commun. 2010, 11450.

(66) (a) Bee, C.; Leclerc, E.; Tius, M. A. Org. Lett. 2003, 5, 4927. (b) Aggarwal, V. K.; Belfield, A. J. Org. Lett. 2003, 5, 5075. (c) Liang, G.; Trauner, D. J. Am. Chem. Soc. 2004, 126, 9544. (d) Janka, M.; He, W.; Haedicke, I. E.; Fronczek, F. R.; Frontier, A. J.; Eisenberg, R. J. Am. Chem. Soc. 2006, 128, 5312. (e) Cao, P.; Deng, C.; Zhou, Y.-Y.; Sun, X.-L.; Zheng, J.-C.; Xie, Z.; Tang, Y. Angew. Chem. Int. Ed. 2010, 49, 4463.

(67) (a) Pellissier, H. Tetrahedron 2005, 61, 6479. (b) Frontier, A. J.; Collinson, C. Tetrahedron 2005, 61, 7577. (c) Tius, M. A. Eur. J. Org. Chem. 2005, 2193. (d) Habermas, K. L.; Denmark, S. E. Org. React. 1994, 45, 1. (e) Denmark, S. E. In Comprehensive Organic Synthesis; Vol. 5; Paquette, L. A., Ed.; Pergamon: Oxford, 1991, 751-784. (f) SantelliRouvier, C.; Santelli, M. Synthesis 1983, 429.

(68) Vaida, T.; Eisenberg, R.; Frontier, A. J. ChemCatChem 2011, 3, 1531 .
(69) Shimada, N.; Stewart, C.; Tius, M. A. Tetrahedron 2011, 67, 5851.

(70) Spencer, W. T. III; Vaidya, T.; Frontier, A. J. Eur. J. Org. Chem. 2013, 3621.

(71) Grant, T. N.; Rieder, C. J.; West, F. G. Chem. Commun. 2009, 5676.

(72) Yaji, K.; Shindo, M. Tetrahedron 2010, 66, 9808.

(73) Williams, D. R.; Robinson, L. A.; Nevill, C. R.; Reddy, J. P. Angew. Chem. Int. Ed. 2007, 46, 915.

(74) Magnus, P.; Freund, W. A.; Moorhead, E. J.; Rainey, T. J. Am. Chem. Soc. 2012, 134, 6140.

(75) Kerr, D. J.; Flynn, B. L. Org. Lett. 2012, 14, 1740.

(76) Lebœuf, D.; Wright, C. M.; Frontier, A. J. Chem. Eur. J. 2013, 19, 4835.

(77) Kerr, D. J.; Miletic, M.; Chaplin, J. H.; White, J. M.; Flynn, B. L. Org. Lett. 2012, 14, 1732.

(78) Flynn, B. L.; Manchala, N.; Krenske, E. H. J. Am. Chem. Soc. 2013, 135, 9156.

(79) Cao, P.; Sun, X.-L.; Zhu, B.-H.; Shen, Q.; Xie, Z.; Tang, Y. Org. Lett. 2009, 11, 3048.

(80) Li, W.-D. Z.; Duo, W.-G.; Zhuang, C.-H. Org. Lett. 2011, 13,3538 .

(81) Spencer, W. T. III; Levin, M. D.; Frontier, A. J. Org. Lett. 2011, 13, 414.

(82) Rautenstrauch, V. J. Org. Chem. 1984, 49, 950.

(83) Nakagawa, D.; Miyashita, M.; Tanino, K. Tetrahedron Lett. 2010, 51, 2771.

(84) Shi, X.; Gorin, D. J.; Toste, F. D. J. Am. Chem. Soc. 2005, 127,5802 .

(85) Kato, K.; Kobayashi, T.; Fujimami, T.; Motodate, S.; Kusakabe, T.; Mochida, T.; Akita, H. Synlett 2008, 1081.

(86) Zhang, L.; Wang, S. J. Am. Chem. Soc. 2006, 128, 1442.

(87) An, S. E.; Jeong, J.; Baskar, B.; Lee, J.; Seo, J.; Rhee, Y. H. Chem. Eur. J. 2009, 15, 11837.

(88) Oh, C. H.; Karmakar, S. J. Org. Chem. 2009, 74, 370.

(89) Tang, J.-M.; Liu, T.-A.; Liu, R.-S. J. Org. Chem. 2008, 73, 8479 .

(90) (a) Prunet, J. Eur. J. Org. Chem. 2011, 3634. (b) Metathesis in Natural Product Synthesis; Cossy, J.; Arseniyadis, S.; Meyer, C., Eds.; Wiley-VCH: Weinheim, 2010.

(c) Monfette, S.; Fogg, D. E. Chem. Rev. 2009, 109, 3783. (d) Majumdar, K. C.; Muhuri, S.; Islam, R.; Chattopadhyay, B. Heterocycles 2009, 78, 1109. (e) Chattopadhyay, S. K.; Karmakar, S.; Biswas, T.; Majumdar, K. C.; Rahaman, H.; Roy, B. Tetrahedron 2007, 63, 3919. (f) Villar, H.; Frings, M.; Bolm, C. Chem. Soc. Rev. 2007, 36, 55. (g) Brown, R. C. D.; Satcharoen, V. Heterocycles 2006, 70, 705. (h) Schmidt, B.; Hermanns, J. Curr. Org. Chem. 2006, 10, 1363. (i) Deiters, A.; Martin, S. F. Chem. Rev. 2004, 104, 2199. (j) Han, S. Y.; Chang, S. In Handbook of Metathesis; Vol. 2; Grubbs, R. H., Ed.; Wiley-VCH: Weinheim, 2010, Chap. 2, 5-127.

(91) Funel, J.-A.; Prunet, J. J. Org. Chem. 2004, 69, 4555.

(92) Dübon, P.; Schelwies, M.; Helmchen, G. Chem. Eur. J. 2008, 14, 6722 .

(93) Miller, A. K.; Hughes, C. C.; Kennedy-Smith, J. J.; Gradl, S. N.; Trauner, D. J. Am. Chem. Soc. 2006, 128, 17057.

(94) (a) Davis, F. A.; Wu, Y. Org. Lett. 2004, 6, 1269. (b) Bullock, K.; Chong, P.; Davies, R.; Elitzin, V.; Hatcher, M.; Jackson, M.; Liu, B.; Patterson, D.; Powers, J.; Salmons, M.; Tabet, E.; Toczko, M. Top. Catal. 2012, 55, 446.

(95) Harmata, M.; Wacharasindhu, S. Org. Lett. 2005, 7, 2563.

(96) Pfeiffer, M. W. B.; Phillips, A. J. J. Am. Chem. Soc. 2005, 127, 5334.

(97) Toueg, J.; Prunet, J. Synlett 2006, 2807.

(98) Diver, S. T.; Guissert, A. J. Chem. Rev. 2004, 104, 1317. 
(99) Ray, D.; Paul, S.; Brahma, S.; Ray, J. K. Tetrahedron Lett. 2007, 48, 8005 .

(100) Ray, D.; Ray, J. K. Org. Lett. 2007, 9, 191.

(101) Ray, D.; Mal, S. K.; Ray, J. K. Synlett 2005, 2135.

(102) Morita, A.; Kuwahara, S. Org. Lett. 2006, 8, 1613.

(103) Tanaka, K.; Fu, G. C. J. Am. Chem. Soc. 2001, 123, 11492.

(104) (a) Tanaka, K.; Fu, G. C. J. Am. Chem. Soc. 2001, 124 , 10296. (b) Tanaka, K.; Fu, G. C. J. Am. Chem. Soc. 2003, 125, 9078 .

(105) Miller, A. K.; Banghart, M. R.; Beaudry, C. M.; Suh, J. M.; Trauner, D. Tetrahedron 2003, 59, 8919.

(106) Kuroda, C.; Honda, S.; Nagura, Y.; Koshio, H.; Shibue, T.; Takeshita, T. Tetrahedron 2004, 60, 319.

(107) Lo, C.-Y.; Lin, C.-C.; Cheng, H.-M.; Liu, R.-S. Org. Lett. 2006, 8,3135 .

(108) Srikrishna, A.; Kumar, P. P. Tetrahedron 2000, 56, 8189.

(109) Pearson, A. J.; Kim, E. H.; Sun, H. Tetrahedron 2010, 66, 4943.

(110) Mehta, G.; Shinde, H. M. Tetrahedron Lett. 2007, 48, 8297.

(111) Andrei, M.; Undheim, K. Tetrahedron: Asymmetry 2004, $15,53$.

(112) Samanta, S.; Yasmin, N.; Kundu, D.; Ray, J. K. Tetrahedron Lett. 2010, 51, 4132.

(113) Kitamura, M.; Chiba, S.; Narasaka, K. Chem. Lett. 2004, 33, 942.

(114) Al-Tel, T. H.; Semreen, M. H.; Voelter, W. Org. Biomol. Chem. 2010, 8, 5375.

(115) Christoffers, J.; Werner, T.; Frey, W.; Baro, A. Chem. Eur. J. 2004, 10, 1042 .

(116) Li, W.-D. Z.; Wang, Y.-Q. Org. Lett. 2003, 5, 2931.

(117) Cho, Y. S.; Carcache, D. A.; Tian, Y.; Li, Y.-M.; Danishefsky, S. J. J. Am. Chem. Soc. 2004, 126, 14358.

(118) Yan, B.; Spilling, C. D. J. Org. Chem. 2008, 73, 5385.

(119) Xu, Y.; McLaughlin, M.; Chen, C.-y.; Reamer, R. A.; Dormer, P. G.; Davies, I. W. J. Org. Chem. 2009, 74, 5100.

(120) Deng, G.; Xu, B.; Wang, J. Tetrahedron 2005, 61, 10811.

(121) Zhuo, X.; Xiang, K.; Zhang, F.-M.; Tu, Y.-Q. J. Org. Chem. 2011, 76, 6918 .

(122) Piancatelli, G.; D'Auria, M.; D’Onofrio, F. Synthesis 1994, 867.

(123) Faza, O. N.; López, C. S.; Álvarez, R.; de Lera, Á. R. Chem. Eur. J. 2004, 10, 4324.

(124) (a) Csákÿ, A. G.; Contreras, C.; Mba, M.; Plumet, J. Synlett 2002, 1451. (b) Csákÿ, A. G.; Mba, M.; Plumet, J. Synlett 2003, 2092.

(125) Harikrishna, M.; Mohan, H. R.; Dubey, P. K.; Subbaraju, G. V. Synth. Commun. 2009, 39, 2763.

(126) Ulbrich, K.; Kreitmeier, P.; Reiser, O. Synlett 2010, 2037.

(127) Yin, B.-L.; Wu, Y.-L.; Lai, J.-Q. Eur. J. Org. Chem. 2009, 2695.

(128) Veits, G. K.; Wenz, D. R.; Read de Alaniz, J. Angew. Chem. Int. Ed. 2010, 49, 9484.

(129) Reddy, B. V. S.; Narasimhulu, G.; Lakshumma, P. S.; Reddy, Y. V.; Yadav, J. S. Tetrahedron Lett. 2012, 53, 1776.

(130) Li, S.-W.; Batey, R. A. Chem. Commun. 2007, 3759.

(131) Wenz, D. R.; Read de Alaniz, J. Org. Lett. 2013, 15, 3250.

(132) Jung, M. E.; Yoo, D. J. Org. Chem. 2007, 72, 8565.

(133) (a) Nunes, J. P. M.; Veiros, L. F.; Vaz, P. D.; Afonso, C. A. M.; Caddick, S. Tetrahedron 2011, 67, 2779. (b) Caddick, S.; Cheung, S.; Doyle, V. E.; Frost, L. M.; Soscia, M. G.; Delisser, V. M.; Williams, M. R. V.; Etheridge, Z. E. Khan S.; Hitchcock, P. B.; Pairaudeau, G.; Vile, S. Tetrahedron 2001, 57, 6295 .
(134) (a) Zou, W.; Shao, H.; Wu, S.-H. Carbohydr. Res. 2004, 339, 2475. (b) Zou, W.; Wang, Z.; Lacroix, E.; Wu, S.-H.; Jennings, H. J. Carbohydr. Res. 2001, 334, 223.

(135) Shipe, W.; Sorensen, E. J. J. Am. Chem. Soc. 2006, 128, 7025.

(136) Matoušová, E.; Růžička, A.; Kuneš, J.; Králová, J.; Pour, M. Chem. Commun. 2011, 47, 9390.

(137) Li, F.; Ding, C.; Wang, M.; Yao, Q.; Zhang, A. J. Org. Chem. 2011, 76, 2820.

(138) Pryde, D. C.; Middleton, D. S.; Stephenson, P. T.; Wainwright, P.; Maddaford, A.; Zhang, X.; Leese, D.; Glen, R.; Hart, J.; Forrest, N.; Guyot, T. Tetrahedron Lett. 2011, 52,6415 .

(139) Kumar, B. S.; Mishra, G. P.; Rao, B. V. Tetrahedron Lett. 2013, 54, 2845.

(140) Petrignet, J.; Prathap, I.; Chandrasekhar, S.; Yadav, J. S.; Grée, R. Angew. Chem. Int. Ed. 2007, 46, 6297.

(141) Hong, F.-T.; Lee, K.-S.; Liao, C.-C. J. Chin. Chem. Soc. 2000, 47,77

(142) Kao, T.-C.; Chuang, G. J.; Liao, C.-C. Angew. Chem. Int. Ed. 2008, 47, 7325 .

(143) (a) Leemans, E.; D'hooghe, M.; De Kimpe, N. Chem. Rev 2011, 111, 3268. (b) Mack, D. J.; Njardarson, J. T. ACS Catal. 2013, 3, 272.

(144) Brown, B.; Hegedus, L. S. J. Org. Chem. 2000, 65, 1865.

(145) Mahuteau-Betzer, F.; Ghosez, L. Tetrahedron 2002, 58, 6991

(146) Gao, F. Y.; Burnell, D. J. J. Org. Chem. 2006, 71, 356.

(147) Coquerel, Y.; Greene, A. E.; Deprés, J.-P. Org. Lett. 2003, 5, 4453

(148) Carret, S.; Deprés, J.-P. Angew. Chem. Int. Ed. 2007, 46, 6870

(149) Varea, T.; Alcalde, A.; de Castillo, C. L.; de Arellano, C. R.; Cossio, F. P.; Asensio, G. J. Org. Chem. 2012, 77, 6327.

(150) (a) Geng, F.; Liu, J.; Paquette, L. A. Org. Lett. 2002, 4, 71. (b) Paquette, L. A.; Geng, F. Org. Lett. 2002, 4, 4547.

(151) Grant, T. N.; West, F. G. J. Am. Chem. Soc. 2006, 128, 9348

(152) Trost, B. M.; Xia, J.; Maulide, N. J. Am. Chem. Soc. 2008, 130, 17258.

(153) Pujanauski, B. G.; Prasad, B. A. B.; Sarpong, R. J. Am. Chem. Soc. 2006, 128, 6787.

(154) Jung, M. E.; Davidov, P. Org. Lett. 2001, 3, 3025.

(155) Matsuda, T.; Tsuboi, T.; Murakami, M. J. Am. Chem. Soc. 2007, 129, 12596.

(156) Mehta, G.; Umarye, J. D. Org. Lett. 2002, 4, 1063.

(157) Iqbal, M.; Duffy, P.; Evans, P.; Cloughley, G.; Allan, B.; Lledó, A.; Verdaguer, X.; Riera, A. Org. Biomol. Chem. 2008, 6, 4649 .

(158) Demuynck, A. L.W.; Levecque, P.; Kidane, A.; Gammon, D. W.; Sickle, E.; Jacobs, P. A.; De Vos, D. E.; Sels, B. F. Adv. Synth. Catal. 2010, 352, 3419.

(159) Shindo, M.; Sato, Y.; Shishido, K. J. Org. Chem. 2001, 66, 7818

(160) Shindo, M.; Sato, Y.; Shishido, K. Tetrahedron Lett. 2002, 43, 5039 .

(161) (a) Kuhn, C.; Skaltsounis, L.; Monneret, C.; Florent, J.-C. Eur. J. Org. Chem. 2003, 2585. (b) Kuhn, C.; Roulland, E.; Madelmont, J.-C.; Monneret, C.; Florent, J.-C. Org. Biomol. Chem. 2004, 2, 2028.

(162) Ballini, R.; Boscia, G.; Fiorini, D.; Gil, M. V.; Petrini, M. Org. Lett. 2001, 3, 1265.

(163) Pal, R.; Clark, R. J.; Manoharan, M.; Alabugin, I. V. J. Org. Chem. 2010, 75, 8689.

(164) Heck, M.-P.; Matt, C.; Wagner, A.; Toupet, L.; Mioskowski, C. Eur. J. Org. Chem. 2010, 966. 